\title{
Genetic polymorphisms in subterranean mammals (Spalax ehrenbergi superspecies) in the Near East revisited: patterns and theory
}

\author{
EVIATAR NEVO*, M. GRAZIA FILIPPUCCI† \& AVIGDOR BEILES \\ Institute of Evolution, University of Haifa, Haifa 31905, Israel and tDipartimento di Biologia, II Universita di Roma, 'Tor \\ Vergata', Via O. Raimondo, 00173 Roma, Italy
}

\begin{abstract}
Allozyme diversity in the superspecies Spalax ehrenbergi has been revisited by studying 36 gene loci in 241 subterranean mole rats from 22 populations and nine chromosomal species, four from Turkey $(2 n=52 \mathrm{E}$ (east), 52W (west), 56 and 58), four from Israel $(2 n=52,54,58$ and 60$)$, and one from Egypt $(2 n=60)$. The following results were indicated. (1) Genetic patterns: 11 of the 36 loci analysed ( 30.5 per cent) were monomorphic across the range, fifteen (41.7 per cent) were weakly polymorphic and the remaining 10 loci (27.8 per cent) were strongly polymorphic. (2) Heterozygosity: the average $H$ was 0.051 , range $0.00-0.098$. In Israel, $H$ increased with aridity and climatic unpredictability towards the northern Negev Desert, and was remarkably high in small steppic semi-isolates and desert isolates. (3) Species discrimination: some of the $S$. ehrenbergi species can be discriminated qualitatively. (4) Genetic distances $(D)$ : between species these values averaged 0.077 , range 0.001-0.269, with the highest $D$ between the ancestor Turkish and descendant Israeli and Egyptian species. The phylogenetic tree supports the Turkish origin of the Israeli Spalax ehrenbergi species, and the recent speciation of the Egyptian Spalax. (5) Genetic diversity is mostly (58 per cent) within populations. (6) Allozyme correlates: allozyme diversity was significantly correlated with the external physical (both climatic and edaphic) and biotic (parasite infection and plant cover) environment. (7) Spatial autocorrelation of allozyme frequencies suggests that migration is not influential. (8) Gametic phase disequilibria were significant in four out of five species tested, and were associated with climatic and edaphic factors. These results support the environmental selection hypothesis of genetic diversity including the niche-width variation hypothesis in space and time. Natural selection appears to play a major role in genetic differentiation of proteins in adaptive radiation and speciation.
\end{abstract}

Keywords: allozyme polymorphisms, mole rats, natural selection, phylogenetic tree, Spalax ehrenbergi, speciation.

\section{Introduction}

Genetic diversity and its differentiation in nature and in subterranean mammals

The structure and determinants of genetic diversity in nature are still debated between the adherents of the neutral (Kimura, 1983) and selective (Nevo, 1978, 1988a; Nevo et al., 1984; Gillespie, 1991) theories of molecular evolution. The evolutionary significance of genetic diversity (heterozygosity) in natural populations of plants and animals has been reviewed using the

\footnotetext{
${ }^{*}$ Correspondence.
}

environmental-genetic correlation methodology at the local, regional, and global levels (Nevo, 1988a). Our results indicated the following: (1) the levels of genetic diversity at all three geographical scales vary nonrandomly among populations, species and higher taxa, and (2) genetic diversity is predictable, primarily by ecological factors. These results corroborated the environmental adaptive theory of genetic diversity and did not fit the prediction of the neutral theory of molecular evolution.

We devoted particular attention to the levels of genetic diversity as a test of the genetic variation niche-width hypothesis (Van Valen, 1965; Soulé \& Stewart, 1970; Nevo, 1978, 1988a) in subterranean 
mammals during the study of the Spalax ehrenbergi superspecies, as an evolutionary model of speciation and adaptation (Nevo, 1991). Our studies involved representatives of the following families: Spalacidae (Nevo \& Shaw, 1972; Nevo \& Cleve, 1978; Nevo et al., 1989, and unpublished data; Savic \& Nevo, 1990); Geomyidae (Nevo et al., 1974); Bathyergidae (Nevo et al., 1987; Burda et al., 1992), and Chrysochloridae (Filippucci et al., 1991). We also reanalysed our and others' data, comparing and contrasting the average level of genetic diversity in subterranean, fossorial and aboveground mammals (Nevo, 1978, 1979, 1983, 1985, 1988a, b; Nevo \& Beiles, 1988; Nevo et al., 1984, 1985a, b, 1990).

Genetic diversity and its ecological correlates in subterranean, fossorial, and aboveground small mammals were reanalysed and verified recently by Nevo et al. (1990) to re-test the environmental theory of genetic diversity, in particular the niche-width variation hypothesis which predicts positive correlation between ecological and phenotypic heterogeneities (Van Valen, 1965). Indeed, the narrow-niche fossorial and subterranean species (Nevo, 1979) displayed significantly lower levels of observed heterozygosity than did small mammalian species living aboveground. This pattern corroborates the genetic variation niche-width hypothesis, or generally the genetic-environmental selection theory of genetic diversity.

\section{The Spalax ehrenbergi evolutionary model}

The $S$. ehrenbergi superspecies of subterranean rodents in Israel consists of four chromosomal species $(2 n=52,54,58$ and 60$)$ displaying progressive stages of late chromosomal speciation. Their adaptive radiation in Israel from Early Pleistocene to recent times is closely associated with the Mediterranean and steppic climates, subterraneity, and a southward environmental gradient of increasing aridity stress towards the Negev Desert. Hence, with distinct climatic diversity: the $2 n=52$ species radiated in the cool, humid northern Upper Galilee Mountains; the $2 n=54$ species in the cool, semi-dry northeastern Golan Heights; the $2 n=58$ species in warm, humid central Israel; and the $2 n=60$ species in warm, dry southern Samaria, Judea, the Negev Mountains, and plains (Fig. 1; see also Figs 2 and 4 in Nevo, 1991).

Observed heterozygosity $H$ in the $S$. ehrenbergi superspecies in Israel, based on 25 allozyme loci, including 17 tissue loci (Nevo \& Shaw, 1972) and eight serum loci (Nevo \& Cleve, 1978) was on average low, ( $H=0.039)$, but it was also positively correlated with climatic unpredictability (Nevo \& Cleve, 1978). It increased southward from mesic to xeric environments as follows: $H=0.035,0.037,0.069$ for the $2 n=52,58$ and 60 species, respectively. A similar trend of increasing $H$ with aridity and climatic unpredictability (or temporally broader ecological-climatic niche) was found in the S. leucodon superspecies in Turkey (Nevo et al., 1989, and unpublished data). The value of $H$ increased from the mesic periphery (in Izmir, $2 n=38$, $H=0.0$ ) to xeric Anatolia (near Ankara, $2 n=62$, $H=0.09)$.

We have earlier shown genetic parallelism of $H$ in 13 unrelated genera ( 21 species, 142 populations, 5474 individuals, each tested, on average, for 27 enzymatic loci) of plants and animals distributed across an aridity gradient in Israel (Nevo \& Beiles, 1988). Heterozygosity, $H$, and genic diversity, $H_{e}$, were positively and, overall, significantly correlated with rainfall variation, i.e. with climatic variation, even in the desert (Nevo \& Beiles, 1989). This corroborates the niche-width variation hypothesis (Van Valen, 1965), or more generally the environmental theory of genetic diversity, at the genetic level (Nevo, 1988a). Note that the S. ehrenbergi superspecies displays underground the same pattern displayed by aboveground organisms of increasing $H$ with climatic unpredictability southward towards the desert (Nevo \& Cleve, 1978).

In this study we tested the $S$. ehrenbergi superspecies for allozymic diversity across a larger range. This included four populations representing four species from southern Turkey; 15 Israeli populations representing four species, which are routinely tested in multidisciplinary studies, and one isolate from Egypt, which was recently identified as a new species in the $S$. ehrenbergi superspecies (Nevo et al., 1991). Previous studies were conducted on genic diversity only in the four Israeli species, but this study focused on five additional species (Turkey and Egypt); it primarily investigated Israeli populations of the four chromosomal species on a different and larger sample size of allozyme loci. Out of the 36 loci studied here, only 10 loci are in common with those tested by Nevo \& Shaw (1972) and only a single locus is in common with those tested by Nevo \& Cleve (1978).

Our main objectives were (1) to revisit genetic diversity patterns in S. ehrenbergi, (2) to correlate them with the environment, to check the prediction of positive correlation with aridity, and (3) to establish a phylogenetic tree based on allozyme diversity. The major question asked was: How much of the variance in allozyme diversity is indeed explicable by ecological (abiotic and biotic) factors? Here, we show that genetic diversity in the $S$. ehrenbergi superspecies is largely non-random and presumably determined to a large extent by natural selection, at both the single and multilocus structures. 
Fig. 1 (a) Distribution of four chromosomal species of Spalax ehrenbergi superspecies in Turkey $(2 n=52 \mathrm{E}$, $52 \mathrm{~W}, 58,56)$. (b) Distribution of four chromosomal species $(2 n=52,54,58$ and 60$)$ of Spalax ehrenbergi superspecies in Israel. Note the hybrid zones separating species and the peripheral semi-isolates and isolates of the $2 n=60$ species. (c) The locality of the new northern Egyptian species $(2 n=60)$ in Burg-El-Arab and ElHammam, near El-Alamein. Numbers in the Israeli range are population numbers that also appear in Table 1.

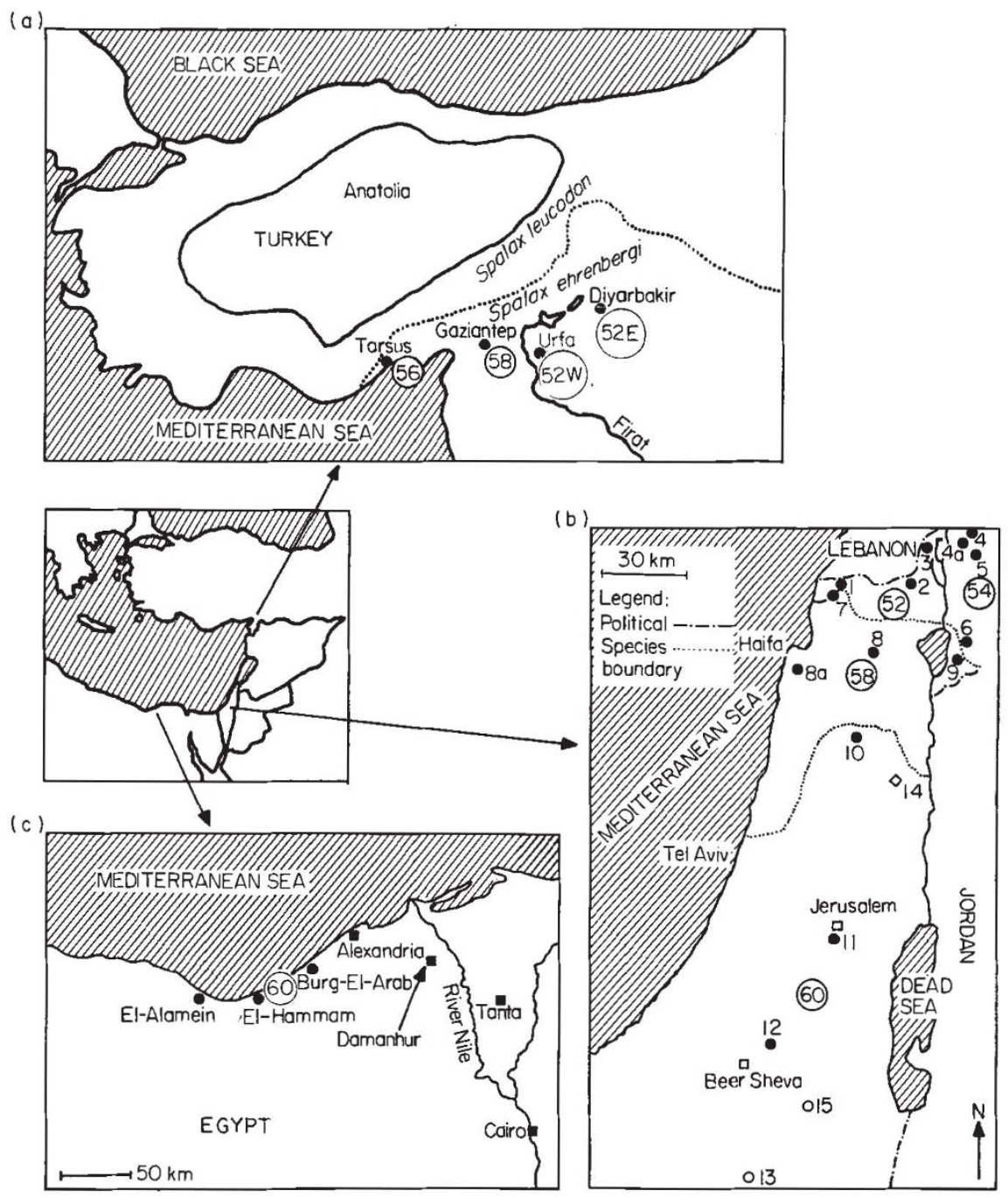

\section{Materials and methods}

\section{Species and populations tested}

Electrophoretic analysis was carried out on 241 subterranean mole rats representing 22 populations of the $S$. ehrenbergi superspecies, comprising four chromosomal species from Israel, four chromosomal species from southern Turkey, and one new species from ElHammam (Burg-El-Arab), near El-Alamein, northern Egypt (Nevo et al., 1991). The chromosomal species $(2 n)$, the collecting sites of the Israeli species and their ecogeographical background appear in Table 1; sample localities and distribution are shown in Fig. 1a, b and c. Tissues of each specimen collected in the wild were preserved in the laboratory at $-80^{\circ} \mathrm{C}$ until processed.

\section{Electrophoretic procedure and loci studied}

Homogenates for electrophoresis were obtained from portions of muscle and kidney tissues crushed in dis- tilled water. Genetic diversity of structural genes encoding for enzymatic and non-enzymatic proteins was assessed using standard horizontal starch-gel electrophoresis (the procedures were those described by Filippucci et al., 1987). All gels were prepared using an 11 per cent suspension of Connaught hydrolyzed starch.

Homogenates obtained from muscle were processed for the following enzymatic proteins: $\alpha$-glycerophosphate dehydrogenase (E.C. 1.1.1.8; $\alpha-G p d h$ ), sorbitol dehydrogenase (E.C. 1.1.1.14; $S d h$ ), lactate dehydrogenase (E.C. 1.1.1.27; $L d h-1$ and $L d h-2$ ), malate dehydrogenase (E.C. 1.1.1.37; $M d h-1$ and $M d h-2$ ), malic enzyme (E.C. 1.1.1.40; $\mathrm{Me}-1$ and $\mathrm{Me}-2$ ), isocitrate dehydrogenase (E.C. 1.1.1.42; Idh- 1 and $I d h-2$ ), 6-phosphogluconate dehydrogenase (E.C. 1.1.1.44; 6Pgdh), glucose-6-phosphate dehydrogenase (E.C. 1.1.1.49; G6pdh), glyceraldehyde-3-phosphate dehydrogenase (E.C. 1.2.1.12; G3pdh), indophenol oxidase (E.C. 1.15.1.1; Ipo), nucleoside phosphorilase (E.C. 2.4.2.1; $N p$ ), glutamate-oxaloacetate transaminase 


\begin{tabular}{|c|c|c|c|c|c|c|c|c|c|c|c|c|c|c|c|c|c|c|c|c|c|c|c|c|c|c|}
\hline \multirow{2}{*}{$\begin{array}{l}\text { Species } \\
\quad \text { No. Population }\end{array}$} & \multirow[b]{2}{*}{ Type* } & \multicolumn{25}{|c|}{ Ecogeographical variables } \\
\hline & & Ln & $\mathrm{Lt}$ & $\mathrm{Al}$ & $\mathrm{Tm}$ & $\mathrm{Ta}$ & $\mathrm{Tj}$ & $\mathrm{Td}$ & Tdd & Trd & $\mathrm{Sh}$ & $\mathrm{Rn}$ & $\mathrm{Rd}$ & $\mathrm{Rv}$ & $\mathrm{Rr}$ & Hu14 & Huan & Dw & Ev & Th & That & So & $\mathrm{Cv}$ & Pl & Dn & $\mathrm{Te}$ \\
\hline \multicolumn{27}{|l|}{$2 n=52$} \\
\hline 1 Maalot & NHZ & 35.27 & 33.00 & 500 & 16.8 & 23.2 & 8.1 & 15.0 & 8.8 & 0 & 63 & 785 & 53 & 31 & 20 & 53 & 64 & 58 & 160 & 15.0 & 20 & 1 & 85 & 3 & 31 & 59 \\
\hline 2 Kerem-Ben-Zimra & $\mathrm{C}$ & 35.47 & 33.03 & 700 & 16.5 & 23.3 & 7.0 & 16.3 & 8.5 & 0 & 85 & 650 & 59 & 32 & 22 & 49 & 60 & 47 & 180 & 4.3 & 0 & 1,3 & 88 & 3 & 40 & 52 \\
\hline 3 Qiryat-Shemona & M & 35.57 & 33.22 & 187 & 19.0 & 26.0 & 9.5 & 16.5 & 10.3 & 50 & 65 & 655 & 60 & 32 & 20 & 49 & 61 & 42 & 200 & 0.0 & 0 & 1,4 & 90 & 5 & 23 & 52 \\
\hline \multicolumn{27}{|l|}{$2 n:=54$} \\
\hline 4 Mt. Hermon & M & 35.73 & 33.30 & 1300 & 12.4 & 20.1 & 3.0 & 17.1 & 5.0 & 0 & 80 & 1450 & 65 & 35 & 20 & 60 & 61 & 60 & 182 & 60.0 & - & 1 & 80 & 1 & 32 & 56 \\
\hline 4a Neve-Ativ & M & 35.70 & 33.30 & & & & & & & & & & & & & & & & & & & & & & & \\
\hline 5 Quneitra & C & 35.83 & 33.12 & 950 & 14.9 & 22.7 & 6.1 & 16.6 & 9.6 & 21 & 71 & 857 & 65 & 33 & 24 & 49 & 61 & 60 & 180 & 33.0 & - & 3 & 40 & 3 & 22 & 54 \\
\hline $6 \quad \mathrm{El}-\mathrm{Al}$ & $\mathrm{NHZ}$ & 35.75 & 32.80 & 370 & 18.7 & 26.0 & 9.6 & 16.4 & 11.1 & 130 & 24 & 464 & 52 & 40 & 26 & 45 & 57 & 50 & 210 & -18.3 & -30 & 3 & 100 & 4 & 39 & 49 \\
\hline \multicolumn{27}{|l|}{$2 n=58$} \\
\hline 7 Kabri & $\mathrm{NHZ}$ & 35.15 & 33.02 & 100 & 20.0 & 26.0 & 10.5 & 13.5 & 10.1 & 0 & 35 & 600 & 50 & 31 & 19 & 58 & 66 & 62 & 150 & -12.7 & -8 & 1,2 & 75 & 5 & 30 & 54 \\
\hline 8 Zippori & C & 35.28 & 32.74 & 250 & 18.5 & 26.0 & 10.4 & 15.4 & 12.0 & 120 & 50 & 500 & 53 & 33 & 22 & 48 & 63 & 70 & 180 & -14.5 & -12 & 2 & 75 & 4 & 26 & 44 \\
\hline 8a Mt. Carmel & $\mathrm{C}$ & 35.04 & 32.69 & 410 & 17.0 & 24.0 & 11.5 & 13.5 & 8.0 & 0 & 45 & 720 & 55 & 24 & 19 & 58 & 69 & 78 & - & - & 5 & - & - & - & - & - \\
\hline 9 Afiq & NHZ,M & 35.70 & 32.77 & 325 & 18.8 & 26.7 & 10.5 & 16.2 & 11.0 & 130 & 24 & 460 & 51 & 40 & 26 & 44 & 57 & 50 & 210 & -18.3 & -30 & 3 & 70 & 4 & 17 & 47 \\
\hline \multicolumn{27}{|l|}{$2 n=60$} \\
\hline 10 Anza & NHZ & 35.22 & 32.35 & 400 & 18.0 & 24.5 & 9.6 & 14.9 & 9.6 & 15 & 65 & 630 & 46 & 35 & 23 & 47 & 62 & 55 & 200 & -6.9 & 0 & 2 & 65 & 3 & 22 & 74 \\
\hline 11 Jerusalem & M & 35.23 & 31.78 & 700 & 17.5 & 23.9 & 8.7 & 15.2 & 9.0 & 0 & 104 & 500 & 42 & 32 & 21 & 51 & 62 & 42 & 190 & -10.3 & -15 & 1 & 45 & 3 & 24 & 91 \\
\hline 12 Lahav & C & 34.87 & 31.38 & 400 & 18.8 & 26.0 & 11.0 & 14.6 & 12.0 & 100 & 60 & 303 & 33 & 35 & 27 & 45 & 58 & 55 & 220 & -32.2 & -45 & 5 & 20 & 2 & 15 & 92 \\
\hline 13 Sede-Boqer & DI & 34.78 & 30.87 & 450 & 19.1 & 25.3 & 9.6 & 15.7 & 13.5 & 100 & - & 91 & 15 & 45 & 29 & 36 & 53 & 70 & 230 & -50.0 & -50 & 5 & - & 6 & - & - \\
\hline 14 Wadi-Fara & SI & - & - & -100 & 22.0 & 29.0 & 13.0 & 16.0 & 12.0 & - & - & 300 & 30 & - & - & 35 & - & - & - & - & -50 & - & - & - & - & - \\
\hline 15 Dimona & DI & 35.03 & 31.03 & 550 & 19.3 & 26.1 & 9.8 & 15.8 & 13.0 & 110 & - & 140 & 21 & 44 & 29 & 37 & 53 & - & 230 & -50.0 & -50 & 5 & - & - & - & - \\
\hline
\end{tabular}

Symbols of ecogeographical variables:

Geographical: Ln, longitude, in decimals; Lt, latitude, in decimals; Al, altitude, in metres.

Temperature: Tm, mean annual temperature $\left({ }^{\circ} \mathrm{C}\right) ; \mathrm{Ta}$, mean August temperature $\left({ }^{\circ} \mathrm{C}\right) ; \mathrm{Tj}$, mean January temperature $\left({ }^{\circ} \mathrm{C}\right) ; \mathrm{Td}$, seasonal temperature difference $\left({ }^{\circ} \mathrm{C}\right)$; Tdd, day-night temperature difference $\left({ }^{\circ} \mathrm{C}\right)$; Trd, mean number of tropical days; Sh, mean number of Sharav days, i.e. hot and dry days.

Water availability: Rn, mean annual rainfall $(\mathrm{mm})$; Rd, mean number of rainy days; Rv, mean interannual variability of rainfall (\%); Rr, mean relative variability of rainfall (\%); Hu 14, mean humidy at 14:00 (\%); Huan, mean annual humidity (\%); Dw, mean number of dew nights in summer; Ev, mean annual evaporation (cm); Th, Thornthwaite's moisture index (our calculation); That, Thornthwaite's moisture index (from Atlas).

Edaphic: So, soil type; 1 , terra rossa; 2, rendzina; 3, basalt; 4, alluvium; 5 , loess

Biotic: Cv, plant cover (\%); Pl, climax plant community (mole rats live only in open spaces or secondary habitats): 1, tragacantic batha; 2, marginal batha; 3 , live oak maquies of $Q$ uercus calliprinos - Pistacia palaestina; 4, Quercus ithaburensis - Styrax officinalis park forest; 5, Ceratonia siliqua - Pistacia lentiscus park forest; 6, Hammada scoparia - Atriplex hamilus desert vegetation; Dn, mole rat population density (animals per $\left.10000 \mathrm{~m}^{2}\right) ; \mathrm{Te}$, territory size $\left(\mathrm{m}^{2}\right)$.

*Type of population: C, central; DI, desert isolate; M, marginal; NHZ, near hybrid zone; SI, semi-isolate. 
(E.C. 2.6.2.1; Got-1 and Got-2), hexokinase (E.C. 2.7.1.1; $H k-1$ and $H k-2$ ), creatine kinase (E.C. 2.7.3.2; $C k$ ), adenylate kinase (E.C. 2.7.4.3; $A d k$ ), phosphoglucomutase (E.C. 2.7.5.1; Pgm-1 and Pgm-2), esterases (E.C. 3.1.1.1; Est-3 and Est-4), aminopeptidase (E.C. 3.4.11.2; $A p-1$ and $A p-2$ ), leucyl aminopeptidase (E.C. 3.4.11.2; Lap), adenosine deaminase (E.C. 3.5.4.4; Ada), aldolase (E.C. 4.1.2.13; Ald), fumarase (E.C. 4.2.1.2; Fum), mannose phosphate isomerase (E.C. 5.3.1.8; $\mathrm{Mpi}$ ), glucose phosphate isomerase (E.C. 5.3.1.9; Pgi), general protein $(P t-1, P t-2$ and $P t-3)$.

Isozymes were numbered in order of decreasing mobility from the most anodal one. Allozymes were designated numerically according to their mobility, relative to the most common allele $(=100$; $<100=$ slower mobility, $>100=$ faster mobility) in the reference population Kerem-Ben-Zimra (K.B.Z.) from Israel.

Intrapopulational genetic diversity was estimated by the following genetic indices: the mean heterozygosity per locus per individual (observed heterozygosity, $H$ ), gene diversity (equal to expected heterozygosity under panmixis, $H_{e}$ ), the proportion of polymorphic loci in the population $(P-1$ per cent or $P-5$ per cent: a locus is considered polymorphic if the frequency of the common allele is not greater than 0.99 or 0.95 , respectively), and the average number of alleles per locus $(A)$. The amount of genetic divergence between populations was estimated with the index of standard genetic distance $(D)$ proposed by Nei $(1972,1978)$. The high number of loci analysed compensates for the small sample size of some populations. Values of heterozygosity and genetic distances are therefore reliable with a reasonable margin of precision (Sarich, 1977; Nei, 1978; Gorman \& Renzi, 1979). Most regional analyses were done excluding Dimona and Wadi-Fara, for which only 28 loci with a few animals were studied. All the other populations were analysed for 36 loci. In Dimona and Wadi-Fara, the following loci were not assessed: $M e-2, H k-1, H k-2, P g m-1$, Est-3, Est-4, Lap, Fum.

\section{Statistical analysis}

Geographical and climatic data for the 17 Israeli populations tested appear in Table 1. These data, derived from the Atlas of Israel (1970) and from multiple year records of the Meteorological Service of Israel, were used in our correlation and multiple regression analyses. Biotic data used in correlations (morphological, physiological and behavioural) were derived from papers cited in Nevo (1991). We used the spss (1990) statistical package for conducting uni- and multivariate analyses. The genetic data were analysed with our own programs and the BIOsys-1 (Release 1.7) package (Swofford \& Selander, 1989). The phylogenetic tree of species was obtained using the Neighbor program of the PHYLIP phylogenetic package (Felsenstein, 1991). For comparison and as an outgroup, we added the nine species of the $S$. leucodon superspecies from Turkey (Nevo et al., 1989, and unpublished data) to the phylogenetic tree. Levels of significance are designated by: $@, P<0.10$; * $P<0.05$; **, $P<0.01$; and ${ }^{* *}, P<0.001$.

\section{Results}

\section{Pattern of variation}

Eleven of the 36 loci analysed ( 30.6 per cent) were monomorphic and fixed for the same allele across the range from southeastern Turkey through Israel to northern Egypt: Ck, Fum, G3pdh, Hk-1, Idh-2, Ipo, Lap, Ldh-2, Pt-1, Pt-2 and Pt-3. The allele frequencies of the polymorphic and/or discriminant loci in the populations analysed are given in Table 2 .

Out of the 25 polymorphic loci, 10 loci were strongly polymorphic (i.e. the most frequent allele was $\leqslant 0.90$ overall frequency) across the range: $A d a, E s t-3$, Est-4, Got-2, Mdh-2, Me-2, Np, Pgi, Sdh, 6Pgdh. All other 15 loci were weakly polymorphic, i.e. $>0.90$ overall frequency.

The numbers of polymorphic loci in the four Israeli species: $2 n=52,54,58$ and 60 were 19, 19, 22 and 23 , respectively. In the Turkish species, $2 n=52 \mathrm{~W}$, $52 \mathrm{E}, 56$ and 58 , the polymorphic loci were $2,3,3$ and 1 , respectively, but sample sizes in Turkey were very low $(N=2-7)$. In the Egyptian small desert isolate of El-Hammam, near El-Alamein, only nine loci were polymorphic.

\section{Hardy-Weinberg deviations}

Significant and almost significant deviations from Hardy-Weinberg $(\mathrm{H}-\mathrm{W})$ equilibrium are given in Table 3. Clearly, there is a significant paucity of heterozygotes in all the 20 deviations reported (sign test $P<0.001)$. Notably, the number of deviations from $\mathrm{H}-\mathrm{W}$ equilibrium increase towards the Negev desert.

\section{Genetic summary}

Levels of genetic diversity within populations are presented in Table 4 . The overall mean heterozygosity $(H)$, based on 36 loci, for all the populations sampled was 0.051 and ranged from 0 (Gaziantep) to 0.098 (Carmel). The lowest values of $H$ were found in the Turkish populations of Gaziantep, Tarsus and Urfa and 


\begin{tabular}{|c|c|c|c|c|c|c|c|c|c|c|c|c|c|c|c|c|c|c|c|c|c|c|c|c|}
\hline Locus & $\begin{array}{c}N: \\
\text { Allele }\end{array}$ & $\begin{array}{c}\text { Diyarbakir } \\
2\end{array}$ & $\begin{array}{c}\text { Urfa } \\
3\end{array}$ & $\begin{array}{c}\text { Gaziantep } \\
2\end{array}$ & $\begin{array}{c}\text { Tarsus } \\
7\end{array}$ & $\begin{array}{c}\text { Maalot } \\
13\end{array}$ & $\begin{array}{c}\text { Zimra } \\
18\end{array}$ & $\begin{array}{c}\text { Shemona } \\
14\end{array}$ & $\begin{array}{l}\text { Hermon } \\
\quad 12\end{array}$ & $\begin{array}{c}\text { Ativ } \\
7\end{array}$ & $\begin{array}{c}\text { Quneitra } \\
10\end{array}$ & $\begin{array}{c}\text { El-Al } \\
12\end{array}$ & $\begin{array}{c}\text { Kabri } \\
14\end{array}$ & $\begin{array}{l}\text { Zippori } \\
15\end{array}$ & $\begin{array}{c}\text { Carmel } \\
15\end{array}$ & $\begin{array}{l}\text { Afiq } \\
11\end{array}$ & $\begin{array}{l}\text { Anza } \\
18\end{array}$ & $\begin{array}{c}\text { Jerusalem } \\
18\end{array}$ & $\begin{array}{l}\text { Lahav } \\
16\end{array}$ & $\begin{array}{l}\text { Boqer } \\
11\end{array}$ & $\begin{array}{c}\text { Dimona } \\
3\end{array}$ & $\begin{array}{c}\text { Fara } \\
3\end{array}$ & $\begin{array}{c}\operatorname{mam} \\
17\end{array}$ & $\begin{array}{c}241 \\
\text { Mean }\end{array}$ \\
\hline \multirow[t]{4}{*}{ Ad } & 95 & 0.0 & 0.0 & 0.0 & 0.0 & 0.038 & 0.028 & 0.0 & 0.0 & 0.0 & 0.0 & 0.042 & 0.036 & 0.067 & 0.0 & 0.0 & 0.0 & 0.0 & 0.0 & 0.0 & 0.0 & 0.0 & 0.059 & 0.017 \\
\hline & 100 & 0.0 & 0.0 & 0.500 & 0.0 & 0.962 & 0.944 & 0.958 & 0.938 & 1.000 & 1.000 & 0.958 & 0.964 & 0.900 & 0.867 & 1.000 & 0.972 & 0.972 & 0.969 & 0.700 & 0.833 & 1.000 & 0.941 & 0.889 \\
\hline & 106 & 1.000 & 1.000 & 0.500 & 1.000 & 0.0 & 0.028 & 0.042 & 0.063 & 0.0 & 0.0 & 0.0 & 0.0 & 0.033 & 0.133 & 0.0 & 0.028 & 0.028 & 0.031 & 0.300 & 0.167 & 0.0 & 0.0 & 0.094 \\
\hline & $H_{p}$ & 0.0 & 0.0 & 0.500 & 0.0 & 0.074 & 0.106 & 0.080 & 0.117 & 0.0 & 0.0 & 0.080 & 0.069 & 0.184 & 0.231 & 0.0 & 0.054 & 0.054 & 0.061 & 0.420 & 0.278 & 0.0 & 0.111 & 0.201 \\
\hline \multirow[t]{3}{*}{$4 d k$} & 100 & 1.000 & 1.000 & 1.000 & 1.000 & 1.000 & 1.000 & 1.000 & 1.000 & 1.000 & 1.000 & 1.000 & 0.857 & 0.923 & 0.767 & 0.773 & 0.667 & 1.000 & 1.000 & 0.786 & 1.000 & 1.000 & 1.000 & 0.925 \\
\hline & 105 & 0.0 & 0.0 & 0.0 & 0.0 & 0.0 & 0.0 & 0.0 & 0.0 & 0.0 & 0.0 & 0.0 & 0.143 & 0.077 & 0.233 & 0.227 & 0.333 & 0.0 & 0.0 & 0.214 & 0.0 & 0.0 & 0.0 & 0.075 \\
\hline & $H_{e}$ & 0.0 & 0.0 & 0.0 & 0.0 & 0.0 & 0.0 & 0.0 & 0.0 & 0.0 & 0.0 & 0.0 & 0.245 & 0.142 & 0.358 & 0.351 & 0.444 & 0.0 & 0.0 & 0.337 & 0.0 & 0.0 & 0.0 & 0.139 \\
\hline \multirow[t]{4}{*}{ Gpdh } & 90 & 1.000 & 1.000 & 0.0 & 0.0 & 0.0 & 0.0 & 0.0 & 0.0 & 0.0 & 0.0 & 0.0 & 0.0 & 0.0 & 0.0 & 0.0 & 0.0 & 0.0 & 0.0 & 0.0 & 0.0 & 0.0 & 0.0 & 0.021 \\
\hline & 100 & 0.0 & 0.0 & 1.000 & 1.000 & 0.962 & 1.000 & 1.000 & 0.958 & 0.786 & 1.000 & 1.000 & 1.000 & 0.933 & 0.967 & 1.000 & 0.972 & 1.0000 & 0.938 & 1.000 & 1.000 & 1.000 & 1.000 & 0.955 \\
\hline & 105 & 0.0 & 0.0 & 0.0 & 0.0 & 0.038 & 0.0 & 0.0 & 0.042 & 0.214 & 0.0 & 0.0 & 0.0 & 0.067 & 0.033 & 0.0 & 0.028 & 0.0 & 0.063 & 0.0 & 0.0 & 0.0 & 0.0 & 0.024 \\
\hline & $H_{e}$ & 0.0 & 0.0 & 0.0 & 0.0 & 0.074 & 0.0 & 0.0 & 0.080 & 0.337 & 0.0 & 0.0 & 0.0 & 0.124 & 0.064 & 0.0 & 0.054 & 0.0 & 0.117 & 0.0 & 0.0 & 0.0 & 0.0 & 0.087 \\
\hline \multirow[t]{3}{*}{$1 / d$} & 100 & 1.000 & 1.000 & 1.000 & 1.000 & 1.000 & 1.000 & 1.000 & 1.000 & 1.000 & 0.889 & 0.955 & 1.000 & 0.923 & 0.933 & 1.000 & 1.000 & 0.933 & 0.893 & 0.857 & 1.000 & 1.000 & 1.000 & 0.966 \\
\hline & 104 & 0.0 & 0.0 & 0.0 & 0.0 & 0.0 & 0.0 & 0.0 & 0.0 & 0.0 & 0.111 & 0.045 & 0.0 & 0.077 & 0.067 & 0.0 & 0.0 & 0.067 & 0.107 & 0.143 & 0.0 & 0.0 & 0.0 & 0.034 \\
\hline & $H_{e}$ & 0.0 & 0.0 & 0.0 & 0.0 & 0.0 & 0.0 & 0.0 & 0.0 & 0.0 & 0.198 & 0.087 & 0.0 & 0.142 & 0.124 & 0.0 & 0.0 & 0.124 & 0.191 & 0.245 & 0.0 & 0.0 & 0.0 & 0.066 \\
\hline \multirow[t]{4}{*}{$4 p-1$} & 95 & 0.0 & 0.333 & 0.0 & 0.0 & 0.0 & 0.0 & 0.0 & 0.0 & 0.0 & 0.0 & 0.0 & 0.0 & 0.0 & 0.0 & 0.0 & 0.0 & 0.0 & 0.0 & 0.0 & 0.0 & 0.0 & 0.0 & 0.005 \\
\hline & 100 & 1.000 & 0.667 & 1.000 & 1.000 & 1.000 & 0.875 & 0.958 & 0.889 & 1.000 & 1.000 & 0.955 & 1.000 & 0.923 & 0.933 & 0.955 & 1.000 & 0.933 & 0.808 & 0.929 & 1.000 & 1,000 & 0.941 & 0.943 \\
\hline & 105 & 0.0 & 0.0 & 0.0 & 0.0 & 0.0 & 0.125 & 0.042 & 0.111 & 0.0 & 0.0 & 0.045 & 0.0 & 0.077 & 0.067 & 0.045 & 0.0 & 0.067 & 0.192 & 0.071 & 0.0 & 0.0 & 0.059 & 0.052 \\
\hline & $H_{e}$ & 0.0 & 0.444 & 0.0 & 0.0 & 0.0 & 0.219 & 0.080 & 0.198 & 0.0 & 0.0 & 0.087 & 0.0 & 0.142 & 0.124 & 0.087 & 0.0 & 0.124 & 0.311 & 0.133 & 0.0 & 0.0 & 0.111 & 0.108 \\
\hline \multirow[t]{4}{*}{$p-2$} & 96 & 0.0 & 0.0 & 0.0 & 0.0 & 0.0 & 0.0 & 0.0 & 0.0 & 0.0 & 0.100 & 0.091 & 0.036 & 0.0 & 0.0 & 0.0 & 0.0 & 0.0 & 0.0 & 0.0 & 0.0 & 0.0 & 0.0 & 0.012 \\
\hline & 100 & 1.000 & 1.000 & 1.000 & 1.000 & 0.917 & 58 & 1.000 & 1.000 & 1.000 & 0.900 & 0.909 & 0.929 & 1.000 & 1.000 & 1.000 & 1.000 & 000 & 1.000 & 1.000 & 333 & .833 & 1.000 & 0.966 \\
\hline & 104 & 0.0 & 0.0 & 0.0 & 0.0 & 0.083 & 0.042 & 0.0 & 0.0 & 0.0 & 0.0 & 0.0 & 0.036 & 0.0 & 0.0 & 0.0 & 0.0 & 0.100 & 0.0 & 0.0 & 0.167 & 0.167 & 0.0 & 0.022 \\
\hline & $H_{e}$ & 0.0 & 0.0 & 0.0 & 0.0 & 0.153 & 0.080 & 0.0 & 0.0 & 0.0 & 0.180 & 0.165 & 0.135 & 0.0 & 0.0 & 0.0 & 0.0 & 0.180 & 0.0 & 0.0 & 0.278 & 0.278 & 0.0 & 0.066 \\
\hline \multirow[t]{5}{*}{$=s t-3$} & 97 & 0.500 & 0.0 & 0.0 & 0.0 & 0.0 & 0.0 & 0.0 & 0.0 & 0.0 & 0.0 & 0.0 & 0.042 & 0.042 & 0.0 & 0.0 & 0.031 & 0.0 & 0.0 & 0.0 & - & - & 0.0 & 0.013 \\
\hline & 100 & 0. & 1.000 & 1.0 & 1.000 & 0.9 & & & & 833 & 1.000 & 0.750 & 0.917 & 0.958 & 0.8 & 0.056 & 0.938 & 14 & 0.682 & 1.000 & - & - & 971 & 0.857 \\
\hline & 103 & 0.250 & 0.0 & 0.0 & 0.0 & & 0.033 & & 0.042 & 0.083 & 0.0 & 0.208 & 0.042 & 0.0 & 0.125 & 0.944 & 0.031 & 0.286 & 0.318 & 0.0 & - & - & 0 & 0.115 \\
\hline & 108 & 0. & 0.0 & 0.0 & 0. & & 0 & & & 0.083 & 0.0 & 0.042 & 0.0 & & 0.0 & 0.0 & 0.0 & & 0.0 & 0.0 & - & - & (1)2. & 0.015 \\
\hline & $H_{e}$ & 0.625 & 0.0 & 0.0 & 0.0 & 0.142 & 0.064 & 0.074 & 0.080 & 0.292 & 0.0 & 0.392 & 0.156 & 0.080 & 0.219 & 0.105 & 0.119 & 0.408 & 0.434 & 0.0 & - & - & 0.057 & 0.252 \\
\hline \multirow[t]{4}{*}{ Est -4} & 95 & 0.0 & 0.0 & 0.0 & 0.0 & 0.063 & 0.19 & & & 0.0 & & 0.0 & 0.188 & 0.100 & 0.167 & & & 0.083 & 0.050 & 0.0 & - & - & 0.143 & 0.085 \\
\hline & 100 & 0.0 & 1.000 & 1.000 & 1.000 & & & & & 000 & 0.938 & 0.850 & 0.813 & 0.900 & 0.833 & 1.667 & 0.900 & 0.7 & 0.850 & 1.000 & - & - & 857 & 0.853 \\
\hline & 105 & 1.000 & 0.0 & 0.0 & 0.0 & & & & 0. & 0.0 & 0. & 0.150 & 0.0 & 0.0 & 0.0 & 0.333 & 0.0 & 0.125 & 0.100 & 0.0 & - & - & & 0.062 \\
\hline & $H_{e}$ & 0.0 & 0.0 & 0.0 & 0.0 & 0.320 & 0.311 & 0.403 & 0.0 & 0.0 & 0.117 & 0.255 & 0.305 & 0.180 & 0.278 & 0.444 & 0.180 & 0.351 & 0.265 & 0.0 & - & - & 0.245 & 0.261 \\
\hline \multirow[t]{4}{*}{ Got-1 } & 90 & 0.0 & 0.167 & 0.0 & 0.0 & 0.0 & 0.0 & 0.0 & 0.0 & 0.0 & 0.0 & 0.0 & 0.0 & 0.0 & 0.0 & 0.0 & 0.0 & 0.0 & 0.0 & 0.0 & 0.0 & 0.0 & 0.0 & 0.002 \\
\hline & 100 & 1.000 & 0.833 & 0.0 & 1.000 & 0. & 00 & & 100 & 929 & 1.000 & 0.958 & 0.929 & 0.967 & 33 & 1.000 & 0.9 & 0.972 & 1.000 & 0.864 & 000 & 1.000 & .000 & 0.956 \\
\hline & 105 & 0.0 & 0.0 & 1.000 & 0.0 & & & & & & 0.0 & & 0.071 & 0.033 & & 0.0 & 0.029 & 0.028 & 0.0 & 0.136 & & 0.0 & 1.0 & 0.042 \\
\hline & $H_{r}$ & 0.0 & 0.278 & 0.0 & 0.0 & 0.074 & 0.0 & 0.0 & 0.0 & 0.133 & 0.0 & 0.080 & 0.133 & 0.064 & 0.278 & 0.0 & 0.057 & 0.054 & 0.0 & 0.236 & 0.0 & 0.0 & 0.0 & 0.084 \\
\hline \multirow[t]{3}{*}{ fot -2} & 100 & 1.000 & 1.0000 & 1.000 & 1.000 & 1.000 & 1.00 & 1000 & 1.00 & 1.000 & 1.000 & 1.000 & 0.179 & 0.300 & 0.300 & 0.0 & 0.333 & 0.139 & 25 & 0.227 & 0.5 & 0.167 & 1.000 & 0.594 \\
\hline & 103 & 0. & 0.0 & 0. & 0. & 0. & 0 & & 0 & 0.0 & 0.0 & 0.0 & 0.821 & 0.7 & 0.7 & 1.000 & 0.667 & 0.8 & 775 & 0.773 & & 0.833 & 0.0 & 0.406 \\
\hline & $H_{v}$ & 0.0 & 0.0 & 0.0 & 0.0 & 0.0 & 0.0 & 0.0 & 0.0 & 0.0 & 0.0 & 0.0 & 0.293 & 0.420 & 0.420 & 0.0 & 0.444 & 0.239 & 0.219 & 0.351 & 0.500 & 0.278 & 0.0 & 0.482 \\
\hline \multirow[t]{3}{*}{ Gopdh } & 100 & 1.000 & 1.000 & 1.000 & 0.929 & 1.000 & 0.9 & & 0.8 & 0.929 & 0.90 & 1.000 & 1.000 & 1.000 & 0.821 & 1.000 & 0.969 & 0.9 & 0.938 & 0.929 & 1.000 & 1.000 & 53 & 0.924 \\
\hline & 105 & 0.0 & 0.0 & & & 0.0 & & & & & & & 0.0 & 0.0 & & & & & & & & 0.0 & & \\
\hline & $H_{e}$ & 0.0 & 0.0 & 0.0 & 0.132 & 0.0 & 0.170 & 0.426 & 0.278 & 0.133 & 0.180 & 0.0 & 0.0 & 0.0 & 0.293 & 0.0 & 0.061 & 0.111 & 0.117 & 0.133 & 0.0 & 0.0 & 0.251 & 0.140 \\
\hline \multirow[t]{3}{*}{$k k \cdot 2$} & 100 & 1.000 & 1.00 & 1.00 & 1.000 & 1.00 & 1.000 & & 1.000 & 1.000 & 1.000 & 1.000 & 1.000 & 1.000 & 1.000 & 0.9 & 1.000 & 0.933 & 1.000 & 1.000 & - & - & 1.000 & 0.990 \\
\hline & 104 & 0.0 & 0.0 & 0.0 & 0.0 & 0.0 & 0.0 & & 0.0 & 0.0 & 0.0 & 0.0 & 0.0 & 0.0 & 0.0 & 0.045 & 0.0 & 0.067 & 0.0 & 0.0 & - & - & 0.0 & 0.010 \\
\hline & $H_{e}$ & 0.0 & 0.0 & 0.0 & 0.0 & 0.0 & 0.0 & 0.074 & 0.0 & 0.0 & 0.0 & 0.0 & 0.0 & 0.0 & 0.0 & 0.087 & 0.0 & 0.124 & 0.0 & 0.0 & - & - & 0.0 & 0.020 \\
\hline
\end{tabular}




\begin{tabular}{|c|c|c|c|c|c|c|c|c|c|c|c|c|c|c|c|c|c|c|c|c|c|c|c|c|}
\hline \multirow[t]{4}{*}{$I d h-I$} & 95 & 0.0 & 0.0 & 0.0 & 0.0 & 0.0 & 0.083 & 0.0 & 0.0 & 0.0 & 0.0 & 0.0 & 0.0 & 0.0 & 0.0 & 0.0 & 0.0 & 0.0 & 0.0 & 0.0 & 0.0 & 0.0 & 0.0 & 0.006 \\
\hline & 100 & 0.0 & 0.667 & 1.000 & 1.000 & 1.000 & 0.917 & 1.000 & 1.000 & 1.000 & 1.000 & 1.000 & 1.000 & 1.000 & 1.000 & 1.000 & 1.000 & 1.000 & 1.000 & 1.000 & 1.000 & 1.000 & 1.000 & 0.981 \\
\hline & 110 & 1.000 & 0.333 & 0.0 & 0.0 & 0.0 & 0.0 & 0.0 & 0.0 & 0.0 & 0.0 & 0.0 & 0.0 & 0.0 & 0.0 & 0.0 & 0.0 & 0.0 & 0.0 & 0.0 & 0.0 & 0.0 & 0.0 & 0.013 \\
\hline & $H_{r}$ & 0.0 & 0.444 & 0.0 & 0.0 & 0.0 & 0.153 & 0.0 & 0.0 & 0.0 & 0.0 & 0.0 & 0.0 & 0.0 & 0.0 & 0.0 & 0.0 & 0.0 & 0.0 & 0.0 & 0.0 & 0.0 & 0.0 & 0.037 \\
\hline \multirow[t]{4}{*}{$L d h-l$} & 95 & 0.0 & 0.0 & 0.0 & 0.429 & 0.192 & 0.0 & 0.0 & 0.292 & 0.0 & 0.0 & 0.0 & 0.0 & 0.0 & 0.0 & 0.0 & 0.0 & 0.028 & 0.063 & 0.0 & 0.0 & 0.0 & 0.0 & 0.045 \\
\hline & 100 & 1.000 & 1.000 & 1.000 & 0.571 & 0.808 & 1.000 & 1.000 & 0.708 & 1.000 & 1.000 & 1.000 & 1.000 & 1.000 & 1.000 & 1.000 & 1.000 & 0.972 & 0.938 & 1.000 & 1.000 & 1.000 & 0.971 & 0.953 \\
\hline & 103 & 0.0 & 0.0 & 0.0 & 0.0 & 0.0 & 0.0 & 0.0 & 0.0 & 0.0 & 0.0 & 0.0 & 0.0 & 0.0 & 0.0 & 0.0 & 0.0 & 0.0 & 0.0 & 0.0 & 0.0 & 0.0 & 0.029 & 0.002 \\
\hline & $H_{r}$ & 0.0 & 0.0 & 0.0 & 0.490 & 0.311 & 0.0 & 0.0 & 0.413 & 0.0 & 0.0 & 0.0 & 0.0 & 0.0 & 0.0 & 0.0 & 0.0 & 0.054 & 0.117 & 0.0 & 0.0 & 0.0 & 0.057 & 0.090 \\
\hline \multirow[t]{3}{*}{$M d h-I$} & 100 & 1.000 & 1.000 & 1.000 & 1.000 & 1.000 & 1.000 & 1.000 & 0.950 & 1.000 & 1.000 & 1.000 & 1.000 & 1.000 & 1.000 & 1.000 & 1.000 & 1.000 & 1.000 & 1.000 & 1.000 & 1.000 & 1.000 & 0.998 \\
\hline & 104 & 0.0 & 0.0 & 0.0 & 0.0 & 0.0 & 0.0 & 0.0 & 0.050 & 0.0 & 0.0 & 0.0 & 0.0 & 0.0 & 0.0 & 0.0 & 0.0 & 0.0 & 0.0 & 0.0 & 0.0 & 0.0 & 0.0 & 0.002 \\
\hline & $H_{p}$ & 0.0 & 0.0 & 0.0 & 0.0 & 0.0 & 0.0 & 0.0 & 0.095 & 0.0 & 0.0 & 0.0 & 0.0 & 0.0 & 0.0 & 0.0 & 0.0 & 0.0 & 0.0 & 0.0 & 0.0 & 0.0 & 0.0 & 0.004 \\
\hline \multirow[t]{3}{*}{$M d h-2$} & 95 & 0.0 & 0.0 & 0.0 & 0.0 & 0.042 & 0.094 & 0.038 & 0.0 & 0.0 & 0.050 & 0.0 & 0.071 & 0.038 & 0.0 & 0.0 & 0.028 & 0.033 & 0.038 & 0.0 & 0.0 & 0.0 & 1.000 & 0.105 \\
\hline & 100 & 1.000 & 1.000 & 1.000 & 1.000 & 0.958 & 0.906 & 0.962 & 1.000 & 1.000 & 0.950 & 1.000 & 0.929 & 0.962 & 1.000 & 1.000 & 0.972 & 0.967 & 0.962 & 1.000 & 1.000 & 1.000 & 0.0 & 0.895 \\
\hline & $H_{e}$ & 0.0 & 0.0 & 0.0 & 0.0 & 0.080 & 0.170 & 0.074 & 0.0 & 0.0 & 0.095 & 0.0 & 0.133 & 0.074 & 0.0 & 0.0 & 0.054 & 0.064 & 0.074 & 0.0 & 0.0 & 0.0 & 0.0 & 0.188 \\
\hline \multirow[t]{4}{*}{$M e-1$} & 95 & 0.0 & 0.0 & 0.0 & 0.0 & 0.0 & 0.0 & 0.0 & 0.0 & 0.0 & 0.0 & 0.0 & 0.0 & 0.0 & 0.0 & 0.0 & 0.0 & 0.0 & 0.031 & 0.0 & 0.0 & 0.0 & 0.0 & 0.002 \\
\hline & 100 & 1.000 & 1.000 & 1.000 & 1.000 & 0.962 & 0.969 & 1.000 & 1.000 & 1.000 & 1.000 & 0.958 & 0.964 & 0.964 & 0.867 & 0.909 & 0.972 & 0.889 & 0.875 & 0.857 & 0.500 & 0.500 & 1.000 & 0.940 \\
\hline & 105 & 0.0 & 0.0 & 0.0 & 0.0 & 0.038 & 0.031 & 0.0 & 0.0 & 0.0 & 0.0 & 0.042 & 0.036 & 0.036 & 0.133 & 0.091 & 0.028 & 0.111 & 0.094 & 0.143 & 0.500 & 0.500 & 0.0 & 0.058 \\
\hline & $H_{e}$ & 0.0 & 0.0 & 0.0 & 0.0 & 0.074 & 0.061 & 0.0 & 0.0 & 0.0 & 0.0 & 0.080 & 0.069 & 0.069 & 0.231 & 0.165 & 0.054 & 0.198 & 0.225 & 0.245 & 0.500 & 0.500 & 0.0 & 0.113 \\
\hline \multirow[t]{4}{*}{$\mathrm{Me}-2$} & 95 & 0.0 & 0.0 & 0.0 & 0.0 & 0.0 & 0.0 & 0.0 & 0.0 & 0.0 & 0.111 & 0.0 & 0.0 & 0.0 & 0.0 & 0.0 & 0.031 & 0.071 & 0.0 & 0.250 & - & - & 0.0 & 0.015 \\
\hline & 100 & 1.000 & 1.000 & 1.000 & 1.0000 & 0.958 & 0.912 & 0.917 & 0.778 & 1.000 & 0.778 & 0.909 & 0.846 & 0.867 & 0.700 & 0.773 & 0.938 & 0.750 & 0.792 & 0.750 & - & - & 0.833 & 0.861 \\
\hline & 105 & 0.0 & 0.0 & 0.0 & 0.0 & 0.042 & 0.088 & 0.083 & 0.222 & 0.0 & 0.111 & 0.091 & 0.154 & 0.133 & 0.300 & 0.227 & 0.031 & 0.179 & 0.208 & 0.0 & - & - & 0.167 & 0.124 \\
\hline & $H_{e}$. & 0.0 & 0.0 & 0.0 & 0.0 & 0.080 & 0.161 & 0.153 & 0.346 & 0.0 & 0.370 & 0.165 & 0.260 & 0.231 & 0.420 & 0.351 & 0.119 & 0.401 & 0.330 & 0.375 & - & - & 0.278 & 0.243 \\
\hline \multirow[t]{5}{*}{$M p i$} & 85 & 0.0 & 0.0 & 0.0 & 0.786 & 0.0 & 0.0 & 0.0 & 0.0 & 0.0 & 0.0 & 0.0 & 0.0 & 0.0 & 0.0 & 0.0 & 0.0 & 0.0 & 0.0 & 0.0 & 0.0 & 0.0 & 0.0 & 0.023 \\
\hline & 90 & 0.0 & 0.0 & 0.0 & 0.214 & 0.0 & 0.250 & 0.0 & 0.0 & 0.0 & 0.0 & 0.042 & 0.0 & 0.0 & 0.0 & 0.0 & 0.0 & 0.0 & 0.0 & 0.0 & 0.0 & 0.0 & 0.0 & 0.028 \\
\hline & $100^{\circ}$ & 0.0 & 0.0 & 0.0 & 0.0 & 1.000 & 0.750 & 1.000 & 1.000 & 1.000 & 1.000 & 0.958 & 1.000 & 1.000 & 0.967 & 0.909 & 1.000 & 0.972 & 1.000 & 0.929 & 1.000 & 1.000 & 1.000 & 0.909 \\
\hline & 105 & 1.000 & 1.000 & 1.000 & 0.0 & 0.0 & 0.0 & 0.0 & 0.0 & 0.0 & 0.0 & 0.0 & 0.0 & 0.0 & 0.033 & 0.091 & 0.0 & 0.028 & 0.0 & 0.071 & 0.0 & 0.0 & 0.0 & 0.040 \\
\hline & $H_{r}$ & 0.0 & 0.0 & 0.0 & 0.0 & 0.0 & 0.375 & 0.0 & 0.0 & 0.0 & 0.0 & 0.080 & 0.0 & 0.0 & 0.064 & 0.165 & 0.0 & 0.054 & 0.0 & 0.133 & 0.0 & 0.0 & 0.0 & 0.171 \\
\hline \multirow[t]{4}{*}{$N p$} & 95 & 0.0 & 0.0 & 0.0 & 1.000 & 0.0 & 0.0 & 0.0 & 0.0 & 0.0 & 0.0 & 0.0 & 0.0 & 0.0 & 0.0 & 0.0 & 0.0 & 0.0 & 0.0 & 0.0 & 0.0 & 0.0 & 0.0 & 0.029 \\
\hline & 100 & 1.000 & 1.000 & 1.000 & 0.0 & 0.885 & 0.972 & 0.846 & 0.875 & 1.000 & 0.700 & 1.000 & 0.893 & 0.967 & 1.000 & 0.818 & 0.972 & 0.917 & 0.969 & 0.950 & 0.833 & 1.000 & 0.941 & 0.900 \\
\hline & 104 & 0.0 & 0.0 & 0.0 & 0.0 & 0.115 & 0.028 & 0.154 & 0.125 & 0.0 & 0.300 & 0.0 & 0.107 & 0.033 & 0.0 & 0.182 & 0.028 & 0.083 & 0.031 & 0.050 & 0.167 & 0.0 & 0.059 & 0.071 \\
\hline & $H_{*}$ & 0.0 & 0.0 & 0.0 & 0.0 & 0.204 & 0.054 & 0.260 & 0.219 & 0.0 & 0.420 & 0.0 & 0.191 & 0.064 & 0.0 & 0.298 & 0.054 & 0.153 & 0.061 & 0.095 & 0.278 & 0.0 & 0.111 & 0.184 \\
\hline \multirow[t]{3}{*}{$P g i$} & 96 & 0.0 & 0.0 & 0.0 & 0.0 & 0.042 & 0.059 & 0.038 & 0.083 & 0.0 & 0.0 & 0.091 & 0.071 & 0.0 & 0.033 & 1.000 & 0.056 & 0.167 & 0.038 & 0.364 & 0.333 & 0.167 & 0.0 & 0.114 \\
\hline & 100 & 1.000 & 1.000 & 1.000 & 1.000 & 0.958 & 0.941 & 0.962 & 0.917 & 1.000 & 1.000 & 0.909 & 0.929 & 1.000 & 0.967 & 0.0 & 0.944 & 0.833 & 0.962 & 0.636 & 0.667 & 0.833 & 1.000 & 0.886 \\
\hline & $\mathrm{H}_{e}$ & 0.0 & 0.0 & 0.0 & 0.0 & 0.080 & 0.111 & 0.074 & 0.153 & 0.0 & 0.0 & 0.165 & 0.133 & 0.0 & 0.064 & 0.0 & 0.105 & 0.278 & 0.074 & 0.463 & 0.444 & 0.278 & 0.0 & 0.202 \\
\hline \multirow[t]{5}{*}{ Pgm-I } & 90 & 0.0 & 0.0 & 0.0 & 0.0 & 0.0 & 0.0 & 0.0 & 0.0 & 0.0 & 0.0 & 0.0 & 0.0 & 0.033 & 0.0 & 0.0 & 0.0 & 0.056 & 0.0 & 0.0 & - & - & 0.0 & 0.007 \\
\hline & 95 & 0.250 & 0.0 & 0.0 & 0.0 & 0.0 & 0.0 & 0.0 & 0.0 & 0.0 & 0.0 & 0.0 & 0.0 & 0.0 & 0.0 & 0.0 & 0.0 & 0.0 & 0.0 & 0.0 & - & - & 0.0 & 0.002 \\
\hline & 100 & 0.750 & 1.000 & 1.000 & 1.000 & 1.000 & 1.000 & 1.000 & 1.000 & 1.000 & 1.000 & 1.000 & 1.000 & 0.867 & 1.000 & 1.000 & 1.000 & 0.917 & 0.867 & 1.000 & - & - & 1.000 & 0.973 \\
\hline & 105 & 0.0 & 0.0 & 0.0 & 0.0 & 0.0 & 0.0 & 0.0 & 0.0 & 0.0 & 0.0 & 0.0 & 0.0 & 0.100 & 0.0 & 0.0 & 0.0 & 0.028 & 0.133 & 0.0 & - & - & 0.0 & 0.018 \\
\hline & $H_{q}$ & 0.375 & 0.0 & 0.0 & 0.0 & 0.0 & 0.0 & 0.0 & 0.0 & 0.0 & 0.0 & 0.0 & 0.0 & 0.238 & 0.0 & 0.0 & 0.0 & 0.156 & 0.231 & 0.0 & - & - & 0.0 & 0.053 \\
\hline \multirow[t]{3}{*}{$P g m-2$} & 100 & 1.000 & 1.000 & 1.000 & 0.0 & 1.000 & 0.944 & 1.000 & 1.000 & 0.929 & 0.950 & 1.000 & 0.893 & 0.933 & 0.867 & 0.955 & 1.000 & 1.000 & 0.767 & 0.909 & 0.500 & 0.667 & 0.912 & 0.906 \\
\hline & 105 & 0.0 & 0.0 & 0.0 & 1.000 & 0.0 & 0.056 & 0.0 & 0.0 & 0.071 & 0.050 & 0.0 & 0.107 & 0.067 & 0.133 & 0.045 & 0.0 & 0.0 & 0.233 & 0.091 & 0.500 & 0.333 & 0.088 & 0.094 \\
\hline & $H_{\mathrm{e}}$ & 0.0 & 0.0 & 0.0 & 0.0 & 0.0 & 0.105 & 0.0 & 0.0 & 0.133 & 0.095 & 0.0 & 0.191 & 0.124 & 0.231 & 0.087 & 0.0 & 0.0 & 0.358 & 0.165 & 0.500 & 0.444 & 0.161 & 0.170 \\
\hline \multirow[t]{4}{*}{$S d h$} & 95 & 0.0 & 0.0 & 0.0 & 0.0 & 0.045 & 0.083 & 0.0 & 0.188 & 0.0 & 0.083 & 0.050 & 0.222 & 0.273 & 0.500 & 0.125 & 1.000 & 0.357 & 1.000 & 0.833 & 0.833 & 0.833 & 1.000 & 0.301 \\
\hline & 100 & 1.000 & 1.000 & 1.000 & 1.000 & 0.955 & 0.917 & 1.000 & 0.813 & 1.000 & 0.917 & 0.950 & 0.167 & 0.182 & 0.0 & 0.875 & 0.0 & 0.071 & 0.0 & 0.0 & 0.0 & 0.0 & 0.0 & 0.548 \\
\hline & 105 & 0.0 & 0.0 & 0.0 & 0.0 & 0.0 & 0.0 & 0.0 & 0.0 & 0.0 & 0.0 & 0.0 & 0.611 & 0.545 & 0.500 & 0.0 & 0.0 & 0.571 & 0.0 & 0.167 & 0.167 & 0.167 & 0.0 & 0.151 \\
\hline & $H_{e}$ & 0.0 & 0.0 & 0.0 & 0.0 & 0.087 & 0.153 & 0.0 & 0.305 & 0.0 & 0.153 & 0.095 & 0.549 & 0.595 & 0.500 & 0.219 & 0.0 & 0.541 & 0.0 & 0.278 & 0.278 & 0.278 & 0.0 & 0.586 \\
\hline $6 P g d h$ & 95 & 0.0 & 0.0 & 0.0 & 0.0 & 0.0 & 0.0 & 0.0 & 0.0 & 0.0 & 0.0 & 0.0 & 0.821 & 0.467 & 0.633 & 0.0 & 0.528 & 0.583 & 0.719 & 0.214 & 0.500 & 0.500 & 1.000 & 0.343 \\
\hline & 100 & 1.000 & 1.000 & 1,000 & 0.0 & 1.000 & 1.000 & 1.000 & 1.000 & 1.000 & 1.000 & 1.000 & 0.179 & 0.533 & 0.367 & 1.000 & 0.472 & 0.417 & 0.281 & 0.786 & 0.500 & 0.500 & 0.0 & 0.627 \\
\hline & 108 & 0.0 & 0.0 & 0.0 & 1.000 & 0.0 & 0.0 & 0.0 & 0.0 & 0.0 & 0.0 & 0.0 & 0.0 & 0.0 & 0.0 & 0.0 & 0.0 & 0.0 & 0.0 & 0.0 & 0.0 & 0.0 & 0.0 & 0.030 \\
\hline & $H_{e}$ & 0.0 & 0.0 & 0.0 & 0.0 & 0.0 & 0.0 & 0.0 & 0.0 & 0.0 & 0.0 & 0.0 & 0.293 & 0.498 & 0.464 & 0.0 & 0.498 & 0.486 & 0.404 & 0.337 & 0.500 & 0.500 & 0.0 & 0.488 \\
\hline
\end{tabular}

Eleven monomorphic loci: Ck, Fum, G3pdh, Hk-l, Idh-2, Ipo, Lap, Ldh-2, Pt-I, Pt-2, Pt-3. 
Table 3 Deviations from Hardy-Weinberg equilibrium in 16 populations of Spalax ehrenbergi in Israel and Egypt*

\begin{tabular}{|c|c|c|c|c|c|c|c|c|c|c|}
\hline \multirow[b]{3}{*}{ Population } & \multirow{3}{*}{$\begin{array}{l}\text { No. of } \\
\text { polymorphic } \\
\text { loci }\end{array}$} & \multirow[b]{3}{*}{ Locus } & \multicolumn{6}{|c|}{ Genotype frequency $\dagger$} & \multirow{3}{*}{$\begin{array}{c}\text { Heterozygote } \\
\text { excess }(+) \text { or } \\
\text { paucity }(-)\end{array}$} & \multirow{3}{*}{$\begin{array}{c}\text { Exact } \\
\text { probability } \\
\text { test } P P\end{array}$} \\
\hline & & & \multicolumn{3}{|c|}{ Observed } & \multicolumn{3}{|c|}{ Expected } & & \\
\hline & & & AA & $\mathrm{Aa}$ & aa & AA & $\mathrm{Aa}$ & aa & & \\
\hline 1 Maalot & 13 & $A p-1$ & 11 & 0 & 1 & 10.08 & 1.84 & 0.08 & - & 0.043 \\
\hline 2 Kerem-Ben-Zimra & 15 & $M d h-2$ & 14 & 1 & 1 & 13.14 & 2.72 & 0.14 & - & 0.097 \\
\hline 2 Kerem-Ben-Zimra & 15 & $I d h-1$ & 16 & 1 & 1 & 15.13 & 2.75 & 0.13 & - & 0.086 \\
\hline 3 Q. Shemona & 10 & - & & & & & & & & \\
\hline 4 Mt. Hermon & 11 & Ap-1 & 8 & 0 & 1 & 7.11 & 1.78 & 0.11 & - & 0.059 \\
\hline 4a Neve-Ativ & 5 & - & & & & & & & & \\
\hline 5 Quneitra & 9 & - & & & & & & & & \\
\hline 6 El-Al & 12 & $A p-2$ & 10 & 0 & 1 & 9.09 & 1.82 & 0.09 & - & 0.048 \\
\hline 6 El-Al & 12 & $M e-2$ & 10 & 0 & 1 & 9.09 & 1.82 & 0.09 & - & 0.048 \\
\hline 7 Kabri & 15 & $S d h$ & 5 & 1 & 3 & 3.36 & 4.28 & 1.36 & - & 0.032 \\
\hline 7 Kabri & 15 & $M d h-2$ & 13 & 0 & 1 & 12.07 & 1.86 & 0.07 & - & 0.037 \\
\hline 8 Zippori & 17 & $A p-1$ & 12 & 0 & 1 & 11.08 & 1.85 & 0.08 & - & 0.040 \\
\hline 8 Zippori & 17 & Est -4 & 9 & 0 & 1 & 8.10 & 1.80 & 0.10 & - & 0.053 \\
\hline 8 Zippori & 17 & $S d h$ & 5 & 2 & 4 & 3.27 & 5.46 & 2.27 & - & 0.060 \\
\hline 8a Mt. Carmel & 17 & - & & & & & & & & \\
\hline 9 Afiq & 11 & - & & & & & & & & \\
\hline 10 Anza & 14 & - & & & & & & & & \\
\hline 11 Jerusalem & 20 & $M e-2$ & 10 & 1 & 3 & 7.88 & 5.25 & 0.87 & - & 0.007 \\
\hline 11 Jerusalem & 20 & $P g m-1$ & 16 & 1 & 1 & 15.13 & 2.75 & 0.13 & - & 0.086 \\
\hline 11 Jerusalem & 20 & $N p$ & 16 & 1 & 1 & 15.13 & 2.75 & 0.13 & - & 0.086 \\
\hline 12 Lahav & 17 & $\alpha G p d h$ & 15 & 0 & 1 & 14.06 & 1.88 & 0.06 & - & 0.032 \\
\hline 12 Lahav & 17 & $L d h-1$ & 15 & 0 & 1 & 14.06 & 1.88 & 0.06 & - & 0.032 \\
\hline 12 Lahav & 17 & $M e-2$ & 9 & 1 & 2 & 7.52 & 3.96 & 0.52 & - & 0.031 \\
\hline 12 Lahav & 17 & $6 P g d h$ & 10 & 3 & 3 & 8.27 & 6.47 & 1.27 & - & 0.046 \\
\hline 13 Sede-Boqer & 15 & - & & & & & & & & \\
\hline E1 El-Hammam & 9 & $M e-2$ & 12 & 1 & 2 & 10.42 & 4.17 & 0.42 & - & 0.019 \\
\hline E1 El-Hammam & 9 & Pgm-2 & 15 & 1 & 1 & 14.13 & 2.74 & 0.13 & - & 0.091 \\
\hline
\end{tabular}

*All deviations with $P<0.10$ are included in the Table.

$\nmid$ Multiallelic loci are pooled: A, most frequent allele; a, all other alleles.

†Significance is calculated by an exact probability test, analogous to Fisher exact test (Vithayasi, 1973).

the Egyptian population of El-Hammam. The highest values of $H$ were largely found in the steppic and desert populations of Jerusalem, Sede-Boqer, Dimona, and Wadi-Fara. In Israel, $H$ basically increases southwards towards higher aridity and climatically unpredictable regimes. The only exception was the highly diverse population of Mt. Carmel. In some withinspecies comparisons between mesic and more steppic populations, such as those between Maalot and Kerem-Ben-Zimra, the above trend was also realized.

Among Israeli mole rats, the lowest values of heterozygosity were found in species with $2 n=52$ and 54 : $H=0.04$, ranging from 0.033 (Neve-Ativ) to 0.056 (Kerem-Ben-Zimra). The mean value of heterozygosity was higher for species with $2 n=58$ and 60: $H=$ $\sim 0.075$, ranging from 0.058 (Kabri) to 0.098 (Mt. Carmel). The values of heterozygosity for Wadi-Fara and Dimona were very high, corresponding to the values observed for other $2 n=60$ populations from Israel, and are based on only 28 loci and few animals. However, according to Gorman and Renzi (1979), in populations with small sample sizes (Urfa, Diyarbakir, Gaziantep, Wadi-Fara and Dimona) the heterozygosity could vary by less than 2.5 per cent as compared with an estimate based on a larger sample size. Remarkably, the $H$ values of three desert isolates and semi-isolates of species with $2 n=60$ in Israel, with small population sizes (Wadi-Fara, Dimona and Sede-Boqer), were very high $(H=0.071,0.095,0.092$, respectively).

The overall mean proportion of polymorphic loci $(P-5$ per cent) for all the populations was 0.218 and ranged from 0.028 (Gaziantep) to 0.417 (Jerusalem). The overall mean of alleles per locus was $A=1.333$, ranging from 1.028 (Gaziantep) to 1.667 (Jerusalem). 
The highest values of $P$ and $A$ were observed in $2 n=60$ populations from Israel. The observed values of genetic diversity are within the range generally reported for other rodents (Nevo et al., 1990), but are higher than previously reported for $S$. ehrenbergi (Nevo \& Cleve, 1978).

\section{Species discrimination}

The Turkish populations from Urfa and Diyarbakir, characterized by $2 n=52$ [west (W) and east (E) species], showed new alleles, for $\alpha G p d h(90)$ and Idh-I (110), that were never found in the other populations.

Table 4 Summary of genetic variation, based on 36 allozyme loci, in 22 populations of nine species of Spalax ehrenbergi in Turkey, Israel and Egypt; in Dimona and Wadi-Fara only 28 loci were assessed; the means are unweighted

\begin{tabular}{|c|c|c|c|c|c|c|c|c|c|}
\hline \multirow[b]{3}{*}{ Locality } & \multirow[b]{3}{*}{$2 n$} & \multirow{3}{*}{$\begin{array}{l}\text { Sample } \\
\text { size } \\
(N)\end{array}$} & \multirow{3}{*}{$\begin{array}{l}\text { Mean no. of } \\
\text { alleles per } \\
\text { locus }(A)\end{array}$} & \multicolumn{4}{|c|}{ Mean proportion of loci } & \multirow{2}{*}{\multicolumn{2}{|c|}{ Gene diversity }} \\
\hline & & & & \multicolumn{2}{|c|}{$\begin{array}{l}\text { Polymorphic per } \\
\text { population } \\
(P)\end{array}$} & \multicolumn{2}{|c|}{$\begin{array}{c}\text { Heterozygous } \\
\text { per individual } \\
\qquad(H)\end{array}$} & & \\
\hline & & & & $(1 \%)$ & $(5 \%)$ & Mean & S.E. & $\left(H_{e}\right)$ & S.E. \\
\hline \multicolumn{10}{|l|}{ Turkey } \\
\hline 1 Diyarbakir & $52 \mathrm{E}$ & 2 & 1.083 & 0.056 & 0.056 & 0.042 & 0.031 & 0.028 & 0.020 \\
\hline 2 Urfa & $52 \mathrm{~W}$ & 3 & 1.083 & 0.083 & 0.083 & 0.028 & 0.020 & 0.032 & 0.019 \\
\hline 3 Gaziantep & 58 & 2 & 1.028 & 0.028 & 0.028 & 0.0 & 0.0 & 0.014 & 0.014 \\
\hline 4 Tarsus & 56 & 7 & 1.083 & 0.083 & 0.083 & 0.024 & 0.017 & 0.027 & 0.017 \\
\hline \multicolumn{10}{|l|}{ Israel } \\
\hline 1 Maalot & 52 & 13 & 1.389 & 0.361 & 0.139 & 0.039 & 0.011 & 0.049 & 0.014 \\
\hline 2 Kerem-Ben-Zimra & 52 & 18 & 1.444 & 0.417 & 0.306 & 0.056 & 0.015 & 0.064 & 0.016 \\
\hline 3 Qiryat Shemona & 52 & 14 & 1.306 & 0.278 & 0.111 & 0.035 & 0.012 & 0.047 & 0.018 \\
\hline Mean & 52 & & 1.380 & 0.352 & 0.185 & 0.043 & 0.006 & 0.053 & 0.005 \\
\hline Sum & 52 & 45 & & & & & & & \\
\hline 4 Mt. Hermon & 54 & 12 & 1.306 & 0.306 & 0.222 & 0.044 & 0.014 & 0.063 & 0.019 \\
\hline 4a Neve-Ativ & 54 & 7 & 1.167 & 0.139 & 0.139 & 0.033 & 0.016 & 0.029 & 0.013 \\
\hline 5 Quneitra & 54 & 10 & 1.278 & 0.250 & 0.194 & 0.048 & 0.016 & 0.050 & 0.017 \\
\hline 6 El-Al & 54 & 12 & 1.361 & 0.333 & 0.139 & 0.040 & 0.014 & 0.048 & 0.015 \\
\hline Mean & 54 & & 1.278 & 0.257 & 0.174 & 0.041 & 0.003 & 0.048 & 0.007 \\
\hline Sum & 54 & 41 & & & & & & & \\
\hline 7 Kabri & 58 & 14 & 1.500 & 0.417 & 0.361 & 0.058 & 0.015 & 0.088 & 0.022 \\
\hline 8 Zippori & 58 & 15 & 1.556 & 0.472 & 0.333 & 0.076 & 0.021 & 0.094 & 0.024 \\
\hline 8a Mt. Carmel & 58 & 15 & 1.472 & 0.472 & 0.389 & 0.098 & 0.024 & 0.121 & 0.027 \\
\hline 9 Afiq & 58 & 11 & 1.306 & 0.306 & 0.222 & 0.062 & 0.019 & 0.066 & 0.020 \\
\hline Mean & 58 & & 1.459 & 0.417 & 0.326 & 0.074 & 0.009 & 0.092 & 0.011 \\
\hline Sum & 58 & 55 & & & & & & & \\
\hline 10 Anza & 60 & 18 & 1.444 & 0.389 & 0.194 & 0.065 & 0.022 & 0.064 & 0.022 \\
\hline 11 Jerusalem & 60 & 18 & 1.667 & 0.556 & 0.417 & 0.087 & 0.023 & 0.115 & 0.026 \\
\hline 12 Lahav & 60 & 16 & 1.528 & 0.472 & 0.361 & 0.070 & 0.019 & 0.100 & 0.023 \\
\hline 13 Sede-Boqer & 60 & 11 & 1.417 & 0.417 & 0.389 & 0.092 & 0.022 & 0.110 & 0.025 \\
\hline 14 Wadi-Fara & 60 & 3 & 1.250 & 0.250 & 0.250 & 0.071 & 0.026 & 0.091 & \\
\hline 15 Dimona & 60 & 3 & 1.321 & 0.321 & 0.321 & 0.095 & 0.029 & 0.127 & \\
\hline Mean* & 60 & & 1.514 & 0.459 & 0.340 & 0.078 & 0.007 & 0.097 & 0.012 \\
\hline Sum $\dagger$ & 60 & $63(69)$ & & & & & & & \\
\hline \multicolumn{10}{|l|}{ Egypt } \\
\hline 1 El-Hammam & 60 & 17 & 1.250 & 0.250 & 0.194 & 0.029 & 0.010 & 0.038 & 0.013 \\
\hline Grand mean* & & & 1.333 & 0.304 & 0.218 & 0.051 & 0.006 & 0.062 & 0.007 \\
\hline Grand sum† & & $\begin{array}{c}235 \\
(241)\end{array}$ & & & & & & & \\
\hline
\end{tabular}

*The mean is based on all populations analysed for 36 loci. Therefore, Wadi-Fara and Dimona were excluded.

$\dagger$ In parentheses is the sum of animals including Dimona and Wadi-Fara. 
Likewise, a new allele of Pgm-I (95) was found in Diyarbakir and new alleles of $A p-I(95)$ and Got-I (90) in Urfa. Finally, the new alleles $M p i(85)$ and $N p(95)$ were found in Tarsus. The loci Ada and Mpi partly discriminated the Turkish species of Diyarbakir, Urfa, Tarsus and Gaziantep from the Egyptian and Israeli species. The locus Got-1 was fixed for the allele 105 in Gaziantep; this allele was not found in Urfa, Diyarbakir, Tarsus and Egypt and it was rare among the Israeli populations. No locus allowed discrimination of $2 n=52$ from $2 n=54$ species nor of $2 n=58$ from $2 n=60$ species in Israel. Three loci, $(S d h, 6 P d g h$ and $G o t-2$ ) did allow a partial discrimination between the two Israeli pairs of species, i.e. the northern pair $(2 n=52,54)$ from the southern pair $(2 n=58,60)$.

Among Israeli populations of $2 n=58$ species, the Afiq population showed peculiarities at several loci. In $S d h$ the allele 100 showed the highest frequency whereas it was rare in other $2 n=58$ populations. In $6 P g d h$ the allele 100 was fixed in Afiq as well as in all $2 n=52$ and 54 populations, whereas in the $2 n=58$ populations (Kabri, Mt. Carmel and nearly in Zippori) the commonest allele was 95. Also fixed in Afiq was the allele 96 of Pgi, which appeared in low frequency in all other populations. The allele 103 of Est-3 was almost fixed in Afiq, whereas it was rare in other Israeli populations.

The chromosome number of the Egyptian population from El-Hammam, near El-Alamein was $2 n=60$. However, the chromosome morphology was different from the Israeli $2 n=60$ species and it was proposed as a different species based on karyotypes, allozymes and behaviour (Nevo et al., 1991). It could be partly discriminated from the Israeli populations of $2 n=60$ by the following loci: $M d h-2,6 P g d h$, and Got-2.

\section{Genetic distance}

Coefficients of genetic distance, $D$, were calculated for paired comparisons of all 20 populations tested for 36 loci, based on normalized identity of all loci between each of the populations, and corrected for small samples (Nei, 1978). $D$ was averaged for paired comparisons of the nine species and the Israeli isolate, as well as for the two regions, Israel and Turkey. The results are given in Table 5, and the phylogenetic tree summarizing the genetic relationships between the species appears in Fig. 2. The mean value of $D$ across all populations was 0.077 , range $0.001-0.269$. The mean genetic distance between the 16 populations of the five southern species (four Israeli, $2 n=52,54,58$, 60 , and one Egyptian species, $2 n=60$ ) was 0.044 , range $0.001-0.184$. The average $D$ between the Israeli complex and the Egyptian $2 n=60$ species was
0.082 , larger than all values of $D$ among the Israeli species.

The values of intraspecific genetic distances within the Israeli species, $2 n=52,54,58$ and 60 were 0.002 , $0.003,0.002$ (excluding Afiq; with Afiq 0.044) and 0.013 , respectively; between the two northern species $2 n=52$ and $54, D=0.003$ and between the two southern species $2 n=58$ and $60, D=0.028$. Higher values of genetic distance separate the northern from the southern Israeli species, $D=0.058$.

By contrast, the genetic distances among the four Turkish species $2 n=58$ (Gaziantep), $2 n=56$ (Tarsus) $2 n=52 \mathrm{E}$ (Diyarbakir) and $2 n=52 \mathrm{~W}$ (Urfa) were higher: $D=0.135$, ranging from 0.053 to 0.236 . A high mean value was also found between the Turkish and all Israeli plus Egyptian species, $D=0.151$. However, the value between the Turkish $2 n=58$ and Israeli $2 n=52$ and 54 was lower, $D=0.064$ (range, $0.060-0.068$ ). Relatively high values of $D$ were also found between the ecologically marginal $2 n=58$ Afiq population and all other Israeli populations $(D=0.084)$, but less so with $2 n=60(D=0.077)$. Its largest genetic distance was with the Egyptian $2 n=60$ species of Burg-ElArab, $D=0.184$.

\section{Phylogenetic tree}

Based on genetic distance $D$, we constructed the phylogenetic tree for the nine $S$. ehrenbergi and nine $S$. leucodon (Nevo et al., unpublished data) species (Fig. 2). The tree has been constructed by the NeighbourJoining method of Saitou and Nei (1987). The tree unfolds a speciation trend from $S$. leucodon in Turkey to $S$. ehrenbergi in Turkey, then through unknown species in Syria and Lebanon to the Israeli complex and Egypt, and it is supported by additional evidence such as fossils, biogeography, cytogenetics, and other molecular evidence (microcomplement fixation, DNA hybridization, and mtDNA; Nevo, 1991). Additional evidence on allozyme grounds (Nevo et al., unpublished data) has indicated that the $S$. ehrenbergi in Turkey originated from the $S$. leucodon superspecies, where the two superspecies are parapatric. Based on this evidence we used the $2 n=38$ species of $S$. leucodon as an outgroup in the phylogenetic tree, because chromosomally it is the farthest apart in the $S$. leucodon complex.

The phylogenetic tree (Fig. 2) suggests that an ancestor of $2 n=58$ and $2 n=56$ species (Gaziantep and Tarsus) may link $S$. leucodon and $S$. ehrenbergi. Thereafter, the Turkish $2 n=56,2 n=58,2 n=52 \mathrm{~W}$ and $2 n=52 \mathrm{E}$ speciated mainly eastward, and the Israeli complex speciated southwards, first giving rise to the northern species pair $(2 n=52$ and 54$)$, and then to the 
Table 5 Coefficients of genetic distance $(D)$ between populations in (a) two regions of Spalax ehrenbergi: Turkey vs. Israel and Egypt, (b) nine species and an isolate of Israeli $2 n=60$, and (c) 20 populations of Spalax ehrenbergi in Turkey, Israel and Egypt

(a) Between regions

\begin{tabular}{|c|c|c|c|}
\hline Region & Populations & 1 & 2 \\
\hline $\begin{array}{l}1 \text { Turkey } \\
\text { range }\end{array}$ & 4 & $\begin{array}{c}0.135 \\
(0.053-0.236)\end{array}$ & $\begin{array}{c}0.151 \\
(0.060-0.269)\end{array}$ \\
\hline $\begin{array}{l}2 \text { Israel and Egypt } \\
\text { range }\end{array}$ & 16 & & $\begin{array}{c}0.044 \\
(0.001-0.184)\end{array}$ \\
\hline
\end{tabular}

(b) Between species

\begin{tabular}{|c|c|c|c|c|c|c|c|c|c|c|c|}
\hline Species & $2 n$ & Populations & 2 & 3 & 4 & 5 & 6 & 7 & 8 & 9 & 10 \\
\hline 1 Turkey, Diyarbakir & $52 \mathrm{E}$ & 1 & 0.053 & 0.138 & 0.236 & 0.155 & 0.161 & 0.218 & 0.227 & 0.211 & 0.269 \\
\hline 2 Turkey, Urfa & $52 \mathrm{~W}$ & 1 & - & 0.063 & 0.159 & 0.093 & 0.095 & 0.159 & 0.164 & 0.131 & 0.195 \\
\hline 3 Turkey, Gaziantep & 58 & 1 & & - & 0.158 & 0.063 & 0.065 & 0.123 & 0.130 & 0.099 & 0.161 \\
\hline 4 Turkey, Tarsus & 56 & 1 & & & - & 0.147 & 0.149 & 0.200 & 0.201 & 0.183 & 0.215 \\
\hline 5 Israel & 52 & 3 & & & & 0.002 & 0.003 & 0.059 & 0.061 & 0.051 & 0.086 \\
\hline 6 Israel & 54 & 4 & & & & & 0.003 & 0.057 & 0.058 & 0.048 & 0.087 \\
\hline 7 Israel & 58 & 4 & & & & & & 0.044 & 0.028 & 0.028 & 0.092 \\
\hline 8 Israel & 60 & 3 & & & & & & & 0.013 & 0.013 & 0.060 \\
\hline 9 Israel, Isolate & 60 & 1 & & & & & & & & - & 0.076 \\
\hline 10 Egypt, El-Hammam & 60 & 1 & & & & & & & & & - \\
\hline
\end{tabular}

(c) Between 20 populations

\begin{tabular}{|c|c|c|c|c|c|c|c|c|c|c|c|c|c|c|c|c|c|c|c|c|}
\hline & Population & $\mathrm{T} 2$ & T3 & $\mathrm{T} 4$ & 1 & 2 & 3 & 4 & $4 a$ & 5 & 6 & 7 & 8 & $8 \mathrm{a}$ & 9 & 10 & 11 & 12 & 13 & E1 \\
\hline T1 & Diyarbakir, Turkey $2 n=52 \mathrm{E}$ & 0.053 & 0.138 & 0.236 & 0.155 & 0.152 & 0.158 & 0.169 & 0.156 & 0.171 & 0.150 & 0.232 & 0.203 & 0.219 & 0.217 & 0.226 & 0.216 & 0.240 & 0.211 & 0.269 \\
\hline $\mathrm{T} 2$ & Urfa, Turkey $2 n=52 \mathrm{~W}$ & & 0.063 & 0.159 & 0.095 & 0.087 & 0.097 & 0.096 & 0.089 & 0.100 & 0.095 & 0.162 & 0.132 & 0.148 & 0.195 & 0.154 & 0.159 & 0.179 & 0.131 & 0.195 \\
\hline T3 & Gaziantep, Turkey $2 n=58$ & & & 0.158 & 0.063 & 0.060 & 0.067 & 0.068 & 0.061 & 0.068 & 0.062 & 0.123 & 0.101 & 0.109 & 0.160 & 0.120 & 0.123 & 0.148 & 0.099 & 0.161 \\
\hline $\mathrm{T} 4$ & Tarsus, Turkey $2 n=56$ & & & & 0.147 & 0.143 & 0.151 & 0.145 & 0.150 & 0.146 & 0.155 & 0.186 & 0.176 & 0.184 & 0.255 & 0.199 & 0.201 & 0.203 & 0.183 & 0.215 \\
\hline 1 & Maalot $2 n=52$ & & & & & 0.003 & 0.002 & 0.002 & 0.002 & 0.002 & 0.001 & 0.057 & 0.036 & 0.054 & 0.087 & 0.053 & 0.053 & 0.074 & 0.050 & 0.087 \\
\hline 2 & Kerem-Ben-Zimra $2 n=52$ & & & & & & 0.002 & 0.005 & 0.004 & 0.004 & 0.002 & 0.058 & 0.036 & 0.051 & 0.089 & 0.052 & 0.054 & 0.072 & 0.049 & 0.082 \\
\hline 3 & Qiryat-Shemona $2 n=52$ & & & & & & & 0.004 & 0.004 & 0.001 & 0.004 & 0.060 & 0.039 & 0.053 & 0.090 & 0.057 & 0.056 & 0.078 & 0.054 & 0.089 \\
\hline 4 & Mt. Hermon $2 n=54$ & & & & & & & & 0.005 & 0.003 & 0.004 & 0.057 & 0.034 & 0.047 & 0.088 & 0.048 & 0.051 & 0.066 & 0.043 & 0.083 \\
\hline $4 a$ & Neve-Ativ $2 n=54$ & & & & & & & & & 0.004 & 0.001 & 0.059 & 0.037 & 0.054 & 0.088 & 0.055 & 0.056 & 0.075 & 0.053 & 0.092 \\
\hline 5 & Quneitra $2 n=54$ & & & & & & & & & & 0.004 & 0.056 & 0.036 & 0.051 & 0.093 & 0.052 & 0.054 & 0.072 & 0.048 & 0.084 \\
\hline 6 & El-Al $2 n=54$ & & & & & & & & & & & 0.057 & 0.036 & 0.051 & 0.073 & 0.053 & 0.050 & 0.070 & 0.049 & 0.090 \\
\hline 7 & Kabri $2 n=58$ & & & & & & & & & & & & 0.002 & 0.002 & 0.088 & 0.018 & 0.002 & 0.019 & 0.023 & 0.063 \\
\hline 8 & Zippori $2 n=58$ & & & & & & & & & & & & & 0.002 & 0.082 & 0.014 & 0.002 & 0.018 & 0.013 & 0.064 \\
\hline $8 \mathrm{a}$ & Mt. Carmel $2 n=58$ & & & & & & & & & & & & & & 0.087 & 0.009 & 0.002 & 0.011 & 0.010 & 0.058 \\
\hline 9 & Afiq $2 n=58$ & & & & & & & & & & & & & & & 0.091 & 0.062 & 0.086 & 0.067 & 0.184 \\
\hline 10 & Anza $2 n=60$ & & & & & & & & & & & & & & & & 0.017 & 0.010 & 0.007 & 0.052 \\
\hline 11 & Jerusalem $2 n=60$ & & & & & & & & & & & & & & & & & 0.012 & 0.014 & 0.071 \\
\hline 12 & Lahav $2 n=60$ & & & & & & & & & & & & & & & & & & 0.017 & 0.058 \\
\hline 13 & Sede-Boqer, Isolate $2 n=60$ & & & & & & & & & & & & & & & & & & & 0.076 \\
\hline E1 & El-Hammam, Egypt $2 n=60$ & & & & & & & & & & & & & & & & & & & \\
\hline
\end{tabular}




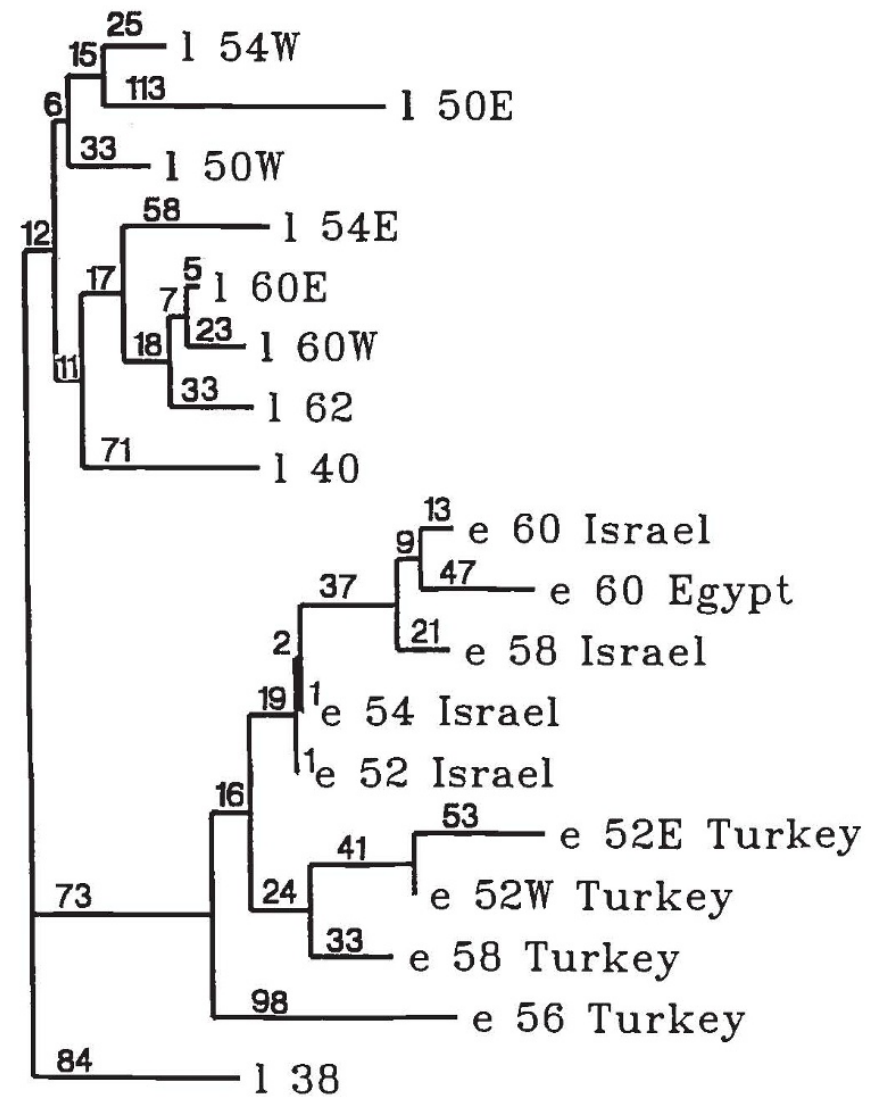

Fig. 2 Phylogenetic tree of nine chromosomal species of the Spalax ehrenbergi superspecies and nine species of the superspecies Spalax leucodon from Turkey. The species with $2 n=38$ of $S$. leucodon in Turkey was used as an outgroup. The tree is based on genetic distances $(D)$, and was constructed by the Neighbor-Joining method of Saitou and Nei (1987). Abbreviations: e, S. ehrenbergi; 1, S. leucodon; E, Eastern species; W, Western species. Numbers represent $2 n$ (diploid chromosome numbers). Branch length is given as $D \times 1000$.

southern species pair $(2 n=58$ and 60$)$. Finally, the new Egyptian species $(2 n=60$, see Nevo et al., 1991) was derived from the Israeli $2 n=60$. The tree does not solve the ancestor-descendant problem in the northern pair $(2 n=52$ and 54$)$, due to the extremely small allozymic genetic distance between them $(D=0.003)$. In the southern species pair it appears that the $2 n=60$ was derived from $2 n=58$ species, although new cytogenetic evidence, (McAllister, B., Greenbaum, I. and Nevo, E., unpublished data), suggests that the $2 n=60$ gave rise to the present $2 n=58$ species.

The estimate of evolutionary divergence times following Nei's (1971) neutral model, using the formula $t=D \times 5 \times 10^{6}$, are as follows. The divergence of $S$. ehrenbergi from $S$. leucodon was 1.2 million years ago; the divergence of the Israeli Spalax from the Turkish origin is 760,000 years ago. The Israeli southern species pair $(2 n=58$ and 60$)$ diverged from the northern species pair $(2 n=52$ and 54) 280,000 years ago, while the $2 n=60$ species separated from the $2 n=58$ species 140,000 years ago. Judging from other molecular data-sets, all these values must be regarded with caution (e.g. Nevo, 1991; Nevo et al., 1993). Selection and other factors (see Nei, 1987 for discussion) may distort these time estimates, and render them unrealistic.

\section{Genetic differentiation within and among populations}

Gene diversity of a subdivided megapopulation $\left(H_{t}\right)$ can be broken down into its components: measurements of the average $\left(D_{s t}\right)$ and the relative $\left(G_{s t}\right)$ degree of gene differentiation among populations, where $H_{s}$ is the mean gene diversity in a population, and $H_{t}$ is the total diversity, and $H_{t}=H_{s}+D_{s t}$ (Nei, 1973). Table 6 summarizes the estimates of the total diversity $\left(H_{t}\right)$ and the proportion expressed between populations $\left(G_{s l}\right)$ for each locus. The average $H$, across all 36 loci of $S$. ehrenbergi in the 15 Israeli populations was 0.12 (range, $0.0-0.57$ ). Seven loci were markedly variable $(>0.20), 15$ loci were near and below the average (0.05-0.20), three loci were weakly polymorphic, and 11 loci were monomorphic. It should be noted that the $H_{t}$, values were distributed discontinuously.

The average relative differentiation $\left(G_{s l}\right)$ among the 20 populations among 25 polymorphic loci was 0.42 (range, 0.05-0.72). In other words, 58 per cent of the allozymic diversity was within populations and 42 per cent was between populations. The highest interpopulation differentiation of $D_{s t}$ was displayed by the following loci: $S d h$ (0.36), Got-2 (0.32), 6Pgdh (0.29), Mdh-2 (0.14), Mpi (0.11), Ada (0.10), Pgi (0.10), Est-3 (0.09), $\mathrm{Np}(0.06)$ and Pgm-2 (0.06).

\section{Environmental correlates with allozyme polymorphisms}

Correlations among environmental variables, and among genetic indices. The environmental variables we used included geographical, ecological (climatic, edaphic and vegetational) and demographic variables (see abbreviations in Table 1). Among the environmental variables water and temperature variables were negatively correlated $\left(\mathrm{Rn}-\mathrm{Tm}, r_{\mathrm{s}}=-0.58\right)$. Temperature means in January and August, and the annual means displayed high correlations $\left(r_{\mathrm{s}}=0.54-0.92\right)$. By contrast, $\mathrm{Td}-\mathrm{Tm}$, for example, displayed low $r_{\mathrm{s}}=-0.10$. Among the genetic indices, the Spearman 
Table 6 Partition of genetic diversity of nine species of the Spalax ehrenbergi superspecies, between and within 20 populations in Turkey, Israel and Egypt, based on 36 loci ( $G_{s t}$ analysis, Nei 1973)

\begin{tabular}{|c|c|c|c|c|c|c|}
\hline Locus & $\begin{array}{c}\text { Alleles } \\
\text { (No.) }\end{array}$ & $\begin{array}{c}\text { Sample } \\
\text { size }\end{array}$ & $H_{t}$ & $H_{s}$ & $D_{s t}$ & $G_{s t}$ \\
\hline$A d a$ & 3 & 227 & 0.2028 & 0.1010 & 0.1018 & 0.5019 \\
\hline$A d k$ & 2 & 215 & 0.1417 & 0.1156 & 0.0261 & 0.1840 \\
\hline$\alpha G p d h$ & 3 & 229 & 0.0886 & 0.0435 & 0.0451 & 0.5089 \\
\hline Ald & 2 & 199 & 0.0676 & 0.0638 & 0.0040 & 0.0594 \\
\hline$A p-1$ & 3 & 213 & 0.1091 & 0.1029 & 0.0081 & 0.0733 \\
\hline$A p-2$ & 3 & 203 & 0.0659 & 0.0542 & 0.0036 & 0.0621 \\
\hline Ck & 1 & 215 & 0.0 & 0.0 & 0.0 & - \\
\hline Est-3 & 4 & 200 & 0.2409 & 0.1610 & 0.0900 & 0.3586 \\
\hline Est-4 & 3 & 153 & 0.2432 & 0.2222 & 0.0392 & 0.1500 \\
\hline Fum & 1 & 131 & 0.0 & 0.0 & 0.0 & - \\
\hline Got-1 & 3 & 233 & 0.0849 & 0.0653 & 0.0210 & 0.2434 \\
\hline Got-2 & 2 & 234 & 0.4837 & 0.1555 & 0.3244 & 0.6760 \\
\hline G6pdh & 2 & 223 & 0.1423 & 0.1313 & 0.0134 & 0.0924 \\
\hline G3pdh & 1 & 174 & 0.0 & 0.0 & 0.0 & - \\
\hline$H k-1$ & 1 & 210 & 0.0 & 0.0 & 0.0 & - \\
\hline$H k-2$ & 2 & 209 & 0.0191 & 0.0181 & 0.0009 & 0.0452 \\
\hline Idh-1 & 3 & 227 & 0.0390 & 0.0180 & 0.0210 & 0.5392 \\
\hline$I d h-2$ & 1 & 145 & 0.0 & 0.0 & 0.0 & - \\
\hline Ipo & 1 & 235 & 0.0 & 0.0 & 0.0 & - \\
\hline Lap & 1 & 168 & 0.0 & 0.0 & 0.0 & - \\
\hline$L d h-1$ & 3 & 230 & 0.0898 & 0.0706 & 0.0207 & 0.2263 \\
\hline$L d h-2$ & 1 & 227 & 0.0 & 0.0 & 0.0 & - \\
\hline$M d h-1$ & 2 & 219 & 0.0045 & 0.0043 & 0.0002 & 0.0478 \\
\hline$M d h-2$ & 2 & 214 & 0.1887 & 0.0528 & 0.1390 & 0.7246 \\
\hline$M e-1$ & 3 & 227 & 0.0924 & 0.0878 & 0.0046 & 0.0497 \\
\hline$M e-2$ & 3 & 197 & 0.2440 & 0.2289 & 0.0151 & 0.0619 \\
\hline$M p i$ & 4 & 230 & 0.1751 & 0.0641 & 0.1110 & 0.6338 \\
\hline$N p$ & 3 & 233 & 0.1856 & 0.1214 & 0.0643 & 0.3462 \\
\hline Pgm-1 & 3 & 219 & 0.0537 & 0.0484 & 0.0053 & 0.0989 \\
\hline Pgm-2 & 2 & 232 & 0.1576 & 0.0976 & 0.0600 & 0.3807 \\
\hline$P g i$ & 2 & 222 & 0.1964 & 0.1008 & 0.0955 & 0.4865 \\
\hline Pt-1 & 1 & 234 & 0.0 & 0.0 & 0.0 & - \\
\hline$P t-2$ & 1 & 235 & 0.0 & 0.0 & 0.0 & - \\
\hline Pt-3 & 1 & 235 & 0.0 & 0.0 & 0.0 & - \\
\hline$S d h$ & 3 & 130 & 0.5724 & 0.2173 & 0.3551 & 0.6205 \\
\hline $6 P d g h$ & 3 & 230 & 0.4866 & 0.1960 & 0.2906 & 0.5971 \\
\hline Mean & & & 0.1223 & 0.0706 & 0.0517 & 0.4225 \\
\hline
\end{tabular}

Abbreviations: $H_{t}$, total gene diversity; $H_{s}$, average gene diversity within populations; $D_{s t}$, average gene diversity between populations; $G_{s t}$, gene diversity between populations, relative to $H_{t}$.

rank correlations were high, ranging between 0.73 $(P<0.001)$ and $0.99(P<0.001)$.

Correlations between genetic indices, allele frequencies and ecogeographical variables in Israel. The Spearman rank correlation matrix between genetic indices, allozymes (strictly local alleles were excluded) and climatic variables appear in Table 7, only for Israel since most climatic parameters in Turkey and Egypt are missing. Out of a matrix of 585 entries, 59 correlations were significant $(P<0.05)$. Thus, a substantial proportion of major alleles displayed ecogeographical correlates above that expected by chance. The genetic indices $\left(A, P-1\right.$ per cent, $P-5$ per cent, $H$ and $\left.H_{e}\right)$ were highly and significantly correlated with longitude, latitude, seasonal temperature difference, number of rainy days and particularly with the first PC of water availability variables representing 52.4 per cent of the variance of 11 variables $\left(r_{\mathrm{s}}=-0.807(P<0.001)-\right.$ $0.534(P<0.05)$, not in Table). The negative correlation with latitude reflects the increase of genetic diversity indices with aridity southward toward the Negev desert. The same is reflected by longitude, as the southern populations are also the western ones, and the northern localities are the most eastern ones (see Ln values in Table 1).

\section{Multiple regression analysis}

Multiple regression (MR) was conducted to find the best predictors of the genetic indices and representative allele frequencies at various polymorphic loci. The results are given in Table 8 for the ecogeographical and the ecological variables separately. A substantial amount of the genetic variation encountered for both overall genetic indices and for the specific allele frequencies was significantly explained by ecogeographical factors $\left(\mathrm{R}^{2}\right.$ values were up to $0.75(P<0.01)$ for the genetic indices $A, P-1$ per cent and $H$, and up to 0.915 $(P<0.001)$ for $M d h-2)$. In particular, the aridity index, as expressed by the number of rainy days and interannual variation in rainfall, or seasonal temperature difference and soil type highly and significantly $\left[\mathrm{R}^{2}=0.70(P<0.01)-0.90(P<0.001)\right]$ explains the variances of all genetic indices and of many of the allele frequencies. Similarly, water availability factors $(R n$, Rd, Dw, Huan and Rv, see Table 1) significantly explain the variances in allele frequency of most polymorphic loci. We conclude that water availability factors, i.e. aridity stress southwards, as well as soil type and plant cover are substantial factors in the genetic differentiation of subterranean mole rats in Israel. We take both the Spearman rank correlations and the multiple regression coefficients as demonstrating selection.

\section{Spatial autocorrelation}

Spatial autocorrelation analysis is a statistical approach for quantifying spatial relations among a set of uni- 
Table 7 Spearman rank correlations $\left(r_{\mathrm{s}}\right)$ of genetic indices and allozyme frequencies with ecogeographical variables in 14 populations of four chromosomal species of the Spalax ehrenbergi superspecies in Israel

\begin{tabular}{|c|c|c|c|c|c|c|c|c|c|c|c|c|c|c|c|c|c|c|}
\hline $\begin{array}{l}\text { Geneti } \\
\text { index/ } \\
\text { allele }\end{array}$ & & $\mathrm{Ln}$ & Lt & $\mathrm{Ta}$ & $\mathrm{Tj}$ & $\mathrm{Td}$ & $\mathrm{Rn}$ & $\mathrm{Rd}$ & $\operatorname{Trd}$ & Ev & Dw & Th & Huan & $\mathrm{Rv}$ & $\mathrm{Rr}$ & Soil types & $\mathrm{Cv}$ & Dn \\
\hline A & & $-0.687^{* *}$ & $-0.623^{*}$ & - & - & $-0.736^{* *}$ & - & $-0.565^{*}$ & - & - & - & - & - & - & - & - & - & - \\
\hline$P-1 \%$ & & $-0.733^{* *}$ & $-0.702^{* *}$ & - & - & $-0.715^{* *}$ & - & $-0.564^{*}$ & - & - & - & - & - & - & - & - & - & - \\
\hline$P-5 \%$ & & $-0.690^{* *}$ & $-0.614^{*}$ & - & - & - & - & - & - & - & - & - & - & - & - & - & - & - \\
\hline$H$ & & $-0.688^{* *}$ & $-0.807^{* * * *}$ & - & $0.553^{*}$ & $-0.582^{*}$ & - & $-0.566^{*}$ & - & - & - & $-0.603^{*}$ & - & - & - & - & $-0.708^{* *}$ & - \\
\hline$H_{e}$ & & $-0.728^{* *}$ & $-0.735^{* * *}$ & - & $0.534^{*}$ & $-0.636^{*}$ & - & $-0.556^{*}$ & - & - & - & $-0.557^{*}$ & - & - & - & - & $-0.670^{*}$ & - \\
\hline Ada & 100 & - & - & - & - & - & - & - & - & - & - & - & - & - & - & - & $0.680^{*}$ & $0.708^{\text {*** }}$ \\
\hline$A d a$ & 106 & - & - & - & - & - & - & - & - & - & - & - & - & - & - & $-0.564 * \mathrm{Bs}$ & - & - \\
\hline$A d k$ & 105 & - & - & - & $0.599^{*}$ & - & - & - & - & - & - & - & - & - & - & - & - & - \\
\hline Ald & 100 & - & $0.556^{*}$ & - & - & - & - & - & - & - & - & - & - & - & - & $-0.649 * \mathrm{Lo}$ & - & - \\
\hline$A p-2$ & 104 & - & - & - & - & - & - & - & $-0.711^{* *}$ & - & - & - & - & $-0.571^{*}$ & - & - & - & - \\
\hline Est -3 & 97 & - & - & - & - & - & - & - & - & - & - & - & - & - & - & $0.741^{* *} \mathrm{Rz}$ & - & - \\
\hline Est -3 & 100 & - & - & - & $-0.585^{*}$ & - & - & - & - & - & - & - & - & - & - & - & - & - \\
\hline Est -3 & 108 & - & - & - & - & - & - & - & - & - & - & - & - & - & - & - & $0.671^{*}$ & - \\
\hline Est -4 & 95 & - & - & - & - & - & - & - & - & $-0.596^{*}$ & - & - & $0.627^{*}$ & $-0.778^{* * * *}$ & $-0.614^{*}$ & - & - & - \\
\hline Est-4 & 100 & - & - & - & - & - & - & - & - & - & $0.635^{*}$ & - & - & - & - & - & - & - \\
\hline Est-4 & 105 & - & - & - & - & - & - & - & - & - & $-0.613^{*}$ & - & - & - & - & - & - & - \\
\hline Got -1 & 100 & - & - & - & - & $0.567^{*}$ & - & - & - & - & $-0.590^{*}$ & - & - & - & - & - & - & - \\
\hline Got -2 & 100 & $0.575^{*}$ & $0.694^{\text {*** }}$ & $-0.553^{*}$ & $-0.716^{* *}$ & $0.573^{*}$ & $0.703 * *$ & $0.744^{* *}$ & - & - & - & $0.699^{* *}$ & - & - & - & - & $0.639^{*}$ & $0.619^{*}$ \\
\hline$H k-2$ & 100 & - & - & - & - & - & - & - & - & - & $0.646^{*}$ & - & - & - & - & - & - & - \\
\hline$M d h-2$ & 100 & - & - & - & - & - & - & - & - & $0.726^{* *}$ & - & - & - & $0.549^{*}$ & - & - & - & - \\
\hline$M e-1$ & 100 & $0.648^{*}$ & $0.816^{* * *}$ & - & $-0.626^{*}$ & $0.570^{*}$ & $0.672^{* *}$ & $0.703^{* *}$ & - & - & - & $0.814^{* * *}$ & - & - & - & $-0.560^{*}$ Lo & - & - \\
\hline$M e-2$ & 105 & - & - & - & - & - & - & - & - & - & - & - & - & - & - & - & $-0.598^{*}$ & - \\
\hline$M p i$ & 100 & - & - & - & - & - & - & - & - & - & - & - & - & - & - & $-0.543^{*} \mathrm{Bs}$ & - & - \\
\hline$P g m-2$ & 100 & $0.577^{*}$ & - & - & $-0.638^{*}$ & - & - & - & - & - & $-0.574^{*}$ & - & - & - & - & - & - & - \\
\hline$S d h$ & 100 & $0.772^{* * *}$ & $0.761^{* * *}$ & - & $-0.538^{*}$ & $0.636^{*}$ & - & $0.630^{*}$ & - & - & - & - & - & - & - & - & $0.731^{* *}$ & - \\
\hline$S d h$ & 105 & $-0.738^{* *}$ & $-0.780^{* * *}$ & - & - & $-0.601^{*}$ & - & $-0.650^{*}$ & - & - & - & - & - & - & - & - & $-0.731^{* *}$ & - \\
\hline 6Pgdh & 100 & $0.796^{* * *}$ & $0.613^{*}$ & - & $-0.671^{* *}$ & $0.843^{* * *}$ & - & $0.602 *$ & - & - & - & - & - & - & - & $0.586 * \mathrm{Bs}$ & - & - \\
\hline
\end{tabular}

Significance: ${ }^{*} P<0.05,{ }^{* *} P<0.01,{ }^{* * *} P<0.001$.

For abbreviations see Table 1 .

Soil type correlations: Bs, with basalt; Rz, with rendzina; Lo, with loess. 
Table 8 Coefficients of multiple regression $\left(\mathbf{R}^{2}\right)$ of allozyme frequencies and their diversity indices in 12 populations of four species of the Spalax ehrenbergi superspecies in Israel, as dependent variables and ecogeographical parameters, including soil type, as independent variables; two populations were excluded due to missing data

\begin{tabular}{|c|c|c|c|c|c|c|c|}
\hline \multicolumn{2}{|c|}{$\begin{array}{c}\text { Dependent } \\
\text { variables }\end{array}$} & \multicolumn{3}{|c|}{ Ecogeographical variables } & \multicolumn{3}{|c|}{ Ecological variables $\dagger$} \\
\hline & & Ln & & & $\mathrm{Rd}$ & Rd Rv & Rd Rv Lo \\
\hline \multirow{2}{*}{\multicolumn{2}{|c|}{$A$}} & $0.604^{* *}$ & - & - & $0.490^{*}$ & $0.688^{* *}$ & $0.747^{* *}$ \\
\hline & & Ln & Ln Ev & & $\mathrm{Rd}$ & Rd Rv & \\
\hline \multirow{2}{*}{\multicolumn{2}{|c|}{$P-1 \%$}} & $0.605^{* *}$ & $0.745^{* *}$ & - & $0.514^{* *}$ & $0.667^{* *}$ & - \\
\hline & & $\mathrm{Ln}$ & & & $\mathrm{Td}$ & $\mathrm{Td} \mathrm{Cv}$ & \\
\hline \multirow{2}{*}{\multicolumn{2}{|c|}{$P-5 \%$}} & $0.372 *$ & - & - & $0.283 @$ & $0.424 @$ & - \\
\hline & & $\mathrm{Lt}$ & Lt Lo & Lt Lo Dw & $\mathrm{Rd}$ & $\mathrm{Rd} \mathrm{Rz}$ & Rd Rz Cv \\
\hline \multirow{2}{*}{\multicolumn{2}{|c|}{$H$}} & $0.501 * *$ & $0.645^{* *}$ & $0.747 * *$ & $0.396^{*}$ & $0.507^{*}$ & $0.606^{*}$ \\
\hline & & $\mathrm{Lt}$ & Lt Lo & & $\mathrm{Rd}$ & $\mathrm{Rd} \mathrm{Hul4}$ & $\mathrm{Rd} \mathrm{Hul} 4 \mathrm{CV}$ \\
\hline \multirow{2}{*}{\multicolumn{2}{|c|}{$H_{e}$}} & $0.548 * *$ & $0.698^{* *}$ & - & $0.478^{*}$ & $0.585^{*}$ & $0.652^{*}$ \\
\hline & & Dw & $\mathrm{Dw} C v$ & Dw Cv Rn & & & \\
\hline \multirow[t]{2}{*}{ Ada } & 95 & $0.254 @$ & $0.540^{*}$ & $0.796^{* *}$ & & & \\
\hline & & $\mathrm{Rz}$ & Rz Sh & Rz Sh Td & & & \\
\hline \multirow{2}{*}{ Adk } & 100 & $0.341^{*}$ & $0.459 @$ & $0.563 @$ & & & \\
\hline & & Dw & Dw Lo & Dw Lo Bs & & & \\
\hline \multirow[t]{2}{*}{$\alpha G p d h$} & 105 & $0.378^{*}$ & $0.624^{*}$ & $0.733^{*}$ & & & \\
\hline & & Lo & $\mathrm{LoCv}$ & Lo Cv Td & & & \\
\hline \multirow[t]{2}{*}{ Ald } & 100 & $0.605^{* *}$ & $0.698^{* *}$ & $0.776^{* *}$ & & & \\
\hline & & Lo & Lo Td & Lo Td Ln & Lo & Lo Td & \\
\hline \multirow[t]{2}{*}{$A p-1$} & 100 & $0.488^{*}$ & $0.625^{*}$ & $0.756^{* *}$ & $0.488^{*}$ & $0.625^{*}$ & - \\
\hline & & $\mathrm{Td}$ & $\mathrm{Td} \mathrm{Rn}$ & Td Rn TR & & & \\
\hline \multirow[t]{2}{*}{$A p-2$} & 104 & $0.306 @$ & $0.498^{*}$ & $0.616^{*}$ & & & \\
\hline & & $\mathrm{Rv}$ & Rv That & & & & \\
\hline \multirow[t]{2}{*}{ Est -3} & 100 & $0.465^{*}$ & $0.570^{*}$ & - & & & \\
\hline & & $\mathrm{Rv}$ & $\operatorname{Rv} \mathrm{Tm}$ & $\mathrm{Rv} \mathrm{Tm} \mathrm{Tj}$ & & & \\
\hline \multirow[t]{2}{*}{ Est -4} & 95 & $0.530^{* *}$ & $0.697^{* *}$ & $0.802^{* *}$ & & & \\
\hline & & $\mathrm{Tm}$ & $\mathrm{Tm} \mathrm{Dw}$ & & & & \\
\hline \multirow[t]{2}{*}{ Est-4 } & 100 & $0.426^{*}$ & $0.662^{* *}$ & - & & & \\
\hline & & $\mathrm{Rv}$ & Rv Dw & & & & \\
\hline \multirow[t]{2}{*}{ Est-4 } & 105 & $0.380^{*}$ & $0.511^{*}$ & - & & & \\
\hline & & $\mathrm{Td}$ & Td Lo & Td Lo Cv & & & \\
\hline \multirow[t]{2}{*}{ Got-1 } & 100 & $0.474^{*}$ & $0.734^{* *}$ & $0.791 * *$ & & & \\
\hline & & Rd & & & & & \\
\hline \multirow[t]{2}{*}{ Got-2 } & 100 & $0.553 * *$ & - & - & & & \\
\hline & & $\mathrm{Td}$ & Td Bs & Td Bs Dw & & & \\
\hline G6pdh & 105 & $\begin{array}{l}0.270 @ \\
\text { Dw }\end{array}$ & $0.523^{*}$ & $0.640^{*}$ & & & \\
\hline$H k-2$ & 100 & $0.461^{*}$ & - & - & & & \\
\hline & & $\mathrm{Rn}$ & $\mathrm{Rn} \mathrm{Rd}$ & & & & \\
\hline$L d h-1$ & 100 & $0.573^{* *}$ & $0.774 * * *$ & - & & & \\
\hline & & $\mathrm{Rv}$ & $\mathrm{Rv} B s$ & Rv Bs Ln & $\mathrm{Rv}$ & Rv Bs & Rv Bs Th \\
\hline$M d h-2$ & 95 & $0.562^{* *}$ & $0.763^{* *}$ & $0.915^{* * *}$ & $0.562^{* *}$ & $0.763^{* *}$ & $0.870 * * *$ \\
\hline & & $\mathrm{Rd}$ & $\mathrm{Rd} \mathrm{Rz}$ & Rd Rz Td & & & \\
\hline$M e-1$ & 100 & $0.751 * * *$ & $0.824 * * *$ & $0.866^{* * *}$ & & & \\
\hline & & $\mathrm{Cv}$ & Cv Ln & Cv Ln Hu14 & $\mathrm{Cv}$ & Cv Td & Cv Td Hu14 \\
\hline$M e-2$ & 100 & $0.411^{*}$ & $0.593^{*}$ & $0.670^{*}$ & $0.411^{*}$ & $0.519^{*}$ & $0.611^{*}$ \\
\hline & & $\mathrm{Cv}$ & Cv Lo & Cv Lo Ln & $\mathrm{Cv}$ & Cv Lo & Cv Lo Td \\
\hline$M e-2$ & 95 & $0.300 @$ & $0.779^{* * *}$ & $0.828 * *$ & $0.300 @$ & $0.779 * * *$ & $0.820^{* *}$ \\
\hline & & Bs & Bs Sh & Bs Sh Ln & Bs & Bs Sh & Bs Sh Al \\
\hline$M p i$ & 100 & $0.383^{*}$ & $0.486^{*}$ & $0.584 @$ & $0.383^{*}$ & $0.486^{*}$ & 0.574@ \\
\hline
\end{tabular}


Table 8 Continued

\begin{tabular}{|c|c|c|c|c|c|c|c|}
\hline \multicolumn{2}{|c|}{$\begin{array}{l}\text { Dependent } \\
\text { variables }\end{array}$} & \multicolumn{3}{|c|}{ Ecogeographical variables } & Stepwise model & \multicolumn{2}{|c|}{ cological variables } \\
\hline & & $\mathrm{Rd}$ & $\mathrm{Rd} \mathrm{Cv}$ & $\mathrm{Rd} \mathrm{Cv} \mathrm{Lt}$ & $\mathrm{Rd}$ & $\mathrm{Rd} \mathrm{Cv}$ & $\mathrm{Rd}$ Cv Tm \\
\hline$N p$ & 100 & $\begin{array}{l}0.279 @ \\
R v\end{array}$ & $0.623^{*}$ & $0.753^{* *}$ & $0.279 @$ & $0.623^{*}$ & $0.707^{*}$ \\
\hline$P g i$ & 100 & $0.367^{*}$ & - & - & & & \\
\hline Pgm-1 & 100 & $\begin{array}{l}\mathrm{Lt} \\
0.507^{* *}\end{array}$ & $\begin{array}{l}\text { Lt Dw } \\
0.652^{* * *}\end{array}$ & - & $\begin{array}{l}\mathrm{Rd} \\
0.371^{*}\end{array}$ & $\begin{array}{l}\text { Rd Dw } \\
0.472 @\end{array}$ & $\begin{array}{l}\text { Rd Dw Td } \\
0.581 @\end{array}$ \\
\hline Pgm-1 & 105 & $\begin{array}{l}\text { Lo } \\
0.592^{* *} \\
\text { Lo }\end{array}$ & $\begin{array}{l}\text { Lo } \mathrm{Rz} \\
0.750^{* *} \\
\text { Lo Ev }\end{array}$ & $\overline{\text { Lo Ev TR }}$ & & & \\
\hline Pgm-2 & 100 & $0.731^{* * *}$ & $0.802^{* * *}$ & $0.871^{* * *}$ & & & \\
\hline & & $\mathrm{Ln}$ & $\mathrm{Ln} \mathrm{Rz}$ & $\mathrm{Ln} \mathrm{Rz} \mathrm{Cv}$ & $\mathrm{Rd}$ & $\mathrm{Rd} \mathrm{Rz}$ & Rd Rz Hu 14 \\
\hline$S d h$ & 100 & $\begin{array}{l}0.641^{* *} \\
\mathrm{Td}\end{array}$ & $\begin{array}{l}0.737^{* *} \\
\text { Td Lt }\end{array}$ & $\begin{array}{l}0.823^{* *} \\
\text { Td Lt Rd }\end{array}$ & $\begin{array}{l}0.558^{* *} \\
\mathrm{Td}\end{array}$ & $\begin{array}{l}0.726^{* *} \\
\text { Td CV }\end{array}$ & $0.866^{* *}$ \\
\hline $6 P g d h$ & 100 & $0.732^{\text {**** }}$ & $0.813^{* * *}$ & $0.851^{* * * * *}$ & $0.732^{* * *}$ & $0.807^{* * *}$ & - \\
\hline
\end{tabular}

Significance: *** $P<0.001$; ** $P<0.01$; * $P<0.05$; $@ P<0.10$.

For abbreviations of ecogeographical variables see Table 1, except soil types:

TR, terra rossa; Rz, rendzina; Bs, basalt; Lo, loess.

fIf in the ecogeographical section only ecological variables were chosen, they were not repeated in this section.

variate data observations. The analysis shows the autocorrelation coefficients as a function of distance between pairs of localities being considered (Sokal \& Oden, $1978 \mathrm{a}, \mathrm{b})$. The analysis was conducted by the SAAP program, release 2.3 (Sokal \& Wartenberg, 1983).

We calculated Moran's $I$ autocorrelation coefficient of alleles in 15 populations across the entire geographical range of the $S$. ehrenbergi superspecies in Israel, involving 36 alleles representing 22 loci. We partitioned the space into six distance classes, so that each class contained almost equal numbers of locality pairs. The following are the main results (Table 9).

(1) Most average autocorrelation coefficients over all tested alleles in most distance groups were low. Note that in each column in Table 9, positive and negative values of low, medium, and sometimes high and significant estimates, sum up into low averages. This pattern indicates that there is no similar pattern across loci in any of the distance groups.

(2) Low order short distance (0-37 and $37-53 \mathrm{~km}$ ) autocorrelations. Positive, negative and no autocorrelations were intermixed across loci. Significant positive autocorrelations were displayed by seven alleles of $S d h, 6 P g d h$, Got-2 and $M e-1$ in the first distance class. Three (in the first) and 10 alleles in the second distance class showed negative autocorrelations $(\leq-0.22)$; only one was significant, but their abundance suggests the existence of an opposite pattern to the expected highest positive autocorrelations in low distances.
(3) Absence of autocorrelations was displayed by several alleles, although they did demonstrate significant correlations in further-distant groups (e.g. $\alpha G p d h$, Ada, Np-1, Got-1, $\mathrm{Me}$-2 and Est-3).

(4) Scattered positive correlations were found at low, medium and high order distances. Some alleles displayed positive autocorrelation in a distant group while negative autocorrelation was observed in closer groups (e.g. Pgi, $A d k$ and $M d h$-2).

(5) Generally, significant negative autocorrelations were more frequent at the high order most distant group.

(6) Six out of the 23 significant correlations appear in intermediate distance classes outside the expected monotonic order. Only two out of 36 alleles show a monotonic decrease in correlations, as expected if autocorrelation is caused by migration.

Five of the above six points negate migration as an explanatory model of the allozyme data, while only point five is in agreement with this model.

\section{Genetic differentiation at the two-locus level: gametic phase disequilibria}

Fifteen significant $(P<0.05)$ gametic phase disequilibria (D) based on two-locus associations were found in four out of five species and in seven out of the 16 populations tested (Table 10). The chromosomal species follow with, in parentheses, their populations 
Table 9 Spatial autocorrelation analysis of 36 alleles representing 22 loci in 15 populations of Spalax ehrenbergi in Israel; the values in the Table are Moran's I autocorrelation coefficient; the expected value is -0.07

\begin{tabular}{|c|c|c|c|c|c|c|c|c|c|c|}
\hline \multirow[b]{3}{*}{ Locus } & \multirow[b]{3}{*}{$\begin{array}{l}\text { No. of pairs: } \\
\text { Allele }\end{array}$} & \multicolumn{6}{|c|}{ Distance group } & & & \\
\hline & & \multirow{2}{*}{$\begin{array}{c}1 \\
0-37 \\
18\end{array}$} & \multirow{2}{*}{$\begin{array}{c}2 \\
37-53 \\
18\end{array}$} & \multirow{2}{*}{$\begin{array}{c}3 \\
53-66 \\
19\end{array}$} & \multirow{2}{*}{$\begin{array}{c}4 \\
66-114 \\
17\end{array}$} & \multirow{2}{*}{$\begin{array}{c}5 \\
114-192 \\
18\end{array}$} & \multirow{2}{*}{$\begin{array}{c}6 \\
192-286(\mathrm{~km}) \\
14\end{array}$} & \multicolumn{3}{|c|}{ Significance summaries } \\
\hline & & & & & & & & \# Significant & \#Monotonic & Intercept \\
\hline Ada & 95 & 0.21 & -0.01 & -0.24 & -0.19 & -0.18 & -0.03 & 0 & 3 & 3 \\
\hline Ada & 100 & 0.04 & 0.05 & -0.02 & -0.18 & 0.01 & $-0.43^{*}$ & 1 & 1 & 4 \\
\hline Ada & 106 & 0.02 & 0.11 & 0.00 & -0.08 & 0.02 & $-0.68^{* * *}$ & 1 & 1 & 4 \\
\hline$A d k$ & 100 & 0.18 & 0.11 & 0.03 & $-0.94^{* * *}$ & 0.15 & 0.05 & 1 & 4 & 4 \\
\hline$\alpha G p d h$ & 100 & -0.11 & -0.19 & -0.09 & -0.02 & -0.05 & 0.07 & 0 & 2 & 1 \\
\hline Ald & 100 & 0.12 & -0.06 & 0.25 & -0.03 & -0.27 & $-0.48^{*}$ & 1 & 2 & 5 \\
\hline$A p-1$ & 100 & -0.27 & 0.16 & 0.06 & 0.05 & -0.19 & -0.30 & 0 & 1 & 1 \\
\hline$A p-2$ & 100 & -0.11 & -0.25 & 0.07 & -0.37 & 0.21 & -0.04 & 0 & 2 & 1 \\
\hline$A p-2$ & 104 & 0.04 & -0.24 & -0.16 & -0.16 & 0.02 & 0.10 & 0 & 2 & 2 \\
\hline Est -3 & 100 & 0.19 & -0.35 & -0.34 & 0.05 & 0.11 & -0.11 & 0 & 2 & 2 \\
\hline Est -3 & 103 & 0.21 & -0.35 & -0.34 & 0.05 & 0.10 & -0.10 & 0 & 2 & 2 \\
\hline Est-3 & 108 & -0.07 & 0.10 & -0.13 & -0.10 & -0.08 & -0.19 & 0 & 1 & 1 \\
\hline Est -4 & 95 & 0.26 & -0.22 & -0.23 & -0.09 & -0.09 & -0.15 & 0 & 3 & 2 \\
\hline Est -4 & 100 & -0.07 & 0.01 & -0.31 & -0.08 & 0.14 & -0.29 & 0 & 1 & 3 \\
\hline Est -4 & 105 & 0.13 & -0.04 & $-0.63^{* *}$ & -0.05 & 0.16 & -0.10 & 1 & 3 & 3 \\
\hline Got-1 & 100 & 0.09 & -0.01 & -0.19 & -0.30 & -0.03 & 0.14 & 0 & 4 & 3 \\
\hline Got-2 & 100 & $0.44^{*}$ & 0.05 & 0.04 & -0.26 & -0.27 & $-0.49^{*}$ & 2 & 6 & 4 \\
\hline$G 6 p d h$ & 100 & 0.21 & -0.26 & $-0.49^{*}$ & 0.21 & -0.01 & -0.05 & 1 & 3 & 2 \\
\hline$L d h-1$ & 100 & -0.35 & -0.05 & 0.15 & -0.09 & -0.09 & 0.11 & 0 & 1 & 1 \\
\hline$M d h-2$ & 100 & 0.09 & $-0.63^{*}$ & 0.10 & 0.03 & 0.04 & -0.14 & 1 & 2 & 2 \\
\hline$M e-1$ & 100 & $0.35^{*}$ & 0.06 & 0.22 & -0.06 & -0.29 & $-0.78^{* * *}$ & 2 & 2 & 5 \\
\hline$M e-1$ & 105 & $0.35^{*}$ & 0.04 & 0.13 & -0.05 & -0.31 & $-0.60^{* *}$ & 2 & 2 & 5 \\
\hline $\mathrm{Me}-2$ & 95 & 0.09 & 0.06 & -0.01 & 0.07 & 0.00 & $-0.69^{* * *}$ & 1 & 3 & 6 \\
\hline $\mathrm{Me}-2$ & 100 & 0.08 & -0.16 & -0.14 & 0.28 & -0.31 & -0.23 & 0 & 2 & 2 \\
\hline $\mathrm{Me}-2$ & 105 & -0.05 & -0.25 & -0.13 & 0.08 & 0.08 & -0.05 & 0 & 2 & 2 \\
\hline$M p i$ & 100 & -0.25 & -0.13 & -0.06 & 0.03 & -0.09 & 0.13 & 0 & 1 & 1 \\
\hline$M p i$ & 105 & 0.08 & -0.17 & -0.25 & 0.14 & -0.10 & -0.05 & 0 & 3 & 2 \\
\hline$N p$ & 100 & -0.13 & -0.01 & 0.14 & -0.34 & 0.05 & -0.17 & 0 & 1 & 1 \\
\hline$P g i$ & 100 & 0.10 & -0.25 & $-0.36^{*}$ & 0.12 & -0.04 & 0.04 & 1 & 3 & 2 \\
\hline Pgm-1 & 100 & 0.01 & -0.06 & 0.12 & 0.05 & -0.36 & -0.25 & 0 & 2 & 5 \\
\hline Pgm-1 & 105 & 0.01 & -0.05 & 0.01 & -0.04 & -0.12 & -0.30 & 0 & 2 & 5 \\
\hline Pgm-2 & 100 & 0.00 & 0.04 & -0.07 & -0.28 & 0.19 & -0.36 & 0 & 1 & 4 \\
\hline$S d h$ & 95 & $0.33^{*}$ & $0.36^{* *}$ & 0.30 & -0.43 & -0.21 & $-0.98^{* * *}$ & 3 & 1 & 4 \\
\hline$S d h$ & 100 & $0.49^{* *}$ & $0.33^{*}$ & 0.10 & -0.42 & -0.40 & $-0.65^{* *}$ & 3 & 4 & 4 \\
\hline$S d h$ & 105 & $0.45^{* *}$ & -0.23 & -0.40 & 0.07 & -0.37 & 0.12 & 1 & 3 & 2 \\
\hline $6 P g d h$ & 100 & $0.42^{*}$ & 0.17 & -0.15 & -0.30 & -0.32 & -0.33 & 1 & 6 & 3 \\
\hline Mean & & 0.10 & -0.06 & -0.08 & -0.10 & -0.08 & -0.23 & \multirow{2}{*}{\multicolumn{3}{|c|}{23 out of $216 I$ values are significant }} \\
\hline Total & & 3.58 & -2.32 & -3.02 & -3.63 & -2.90 & -8.26 & & & \\
\hline
\end{tabular}


Table 10 Gametic phase disequilibria, D, with $P<0.05$ by one-tailed Fisher exact test, found in 16 populations of Spalax ehrenbergi in Israel and Egypt

\begin{tabular}{|c|c|c|c|c|c|c|c|c|}
\hline \multirow[b]{2}{*}{ Population } & \multicolumn{2}{|c|}{ Loci } & \multirow[b]{2}{*}{$\mathrm{D}$} & \multicolumn{4}{|c|}{ Genotype frequency } & \multirow{2}{*}{$\begin{array}{c}\text { Fisher exact } \\
\text { one-tail test } \\
P\end{array}$} \\
\hline & 1 & 2 & & ‘1 1 ’ & ' $10 ’$ & '01' & ‘ 00 ’ & \\
\hline 4 Mt. Hermon & $A p-1$ & $N p$ & 0.093 & 15 & 1 & 0 & 2 & 0.020 \\
\hline $4 \mathrm{Mt}$. Hermon & $M e-2$ & $S d h$ & 0.160 & 8 & 0 & 0 & 2 & 0.022 \\
\hline 6 El-Al & $A p-2$ & Est-3 & 0.070 & 17 & 0 & 3 & 2 & 0.043 \\
\hline 7 Kabri & $M d h-2$ & Got-2 & 0.059 & 3 & 2 & 23 & 0 & 0.027 \\
\hline 7 Kabri & $M d h-2$ & $N p$ & 0.064 & 25 & 0 & 1 & 2 & 0.008 \\
\hline 11 Jerusalem & $A p-2$ & $M e-2$ & 0.080 & 21 & 0 & 4 & 3 & 0.011 \\
\hline 11 Jerusalem & $M e-1$ & $A p-2$ & 0.057 & 26 & 1 & 1 & 2 & 0.020 \\
\hline 11 Jerusalem & $M e-1$ & $M e-2$ & 0.080 & 21 & 0 & 4 & 3 & 0.011 \\
\hline 12 Lahav & $\alpha G p d h$ & Ald & 0.074 & 18 & 0 & 2 & 2 & 0.026 \\
\hline 12 Lahav & $\alpha G p d h$ & $A p-1$ & 0.062 & 21 & 0 & 3 & 2 & 0.031 \\
\hline 12 Lahav & $\alpha G p d h$ & $M e-2$ & 0.066 & 19 & 0 & 3 & 2 & 0.036 \\
\hline 12 Lahav & Ald & $L d h-1$ & 0.074 & 18 & 0 & 2 & 2 & 0.026 \\
\hline 12 Lahav & $A p-1$ & $M e-2$ & 0.090 & 18 & 1 & 2 & 3 & 0.018 \\
\hline 13 Sede-Boqer & Got-1 & Got-2 & 0.073 & 2 & 2 & 17 & 0 & 0.029 \\
\hline E1 El-Hammam & $P g m-2$ & $M e-2$ & 0.057 & 26 & 0 & 2 & 2 & 0.014 \\
\hline
\end{tabular}

and the number of significant disequilibria. Israel: $2 n=54$ (Hermon, 2; El-Al, 1); $2 n=58$ (Kabri, 2); $2 n=60$ (Jerusalem, 3; Lahav, 5; the Sede-Boqer small desert isolate, 1); and the Egyptian $2 n=60$ new species (near El-Alamein, 1). Notably, most significant $\mathrm{D}$ values occur in the $2 n=60$ species, which lives under the highest climatic (aridity) stress.

The 15 significant $\mathrm{D}$ values are nearly 3 per cent of all pairwise combinations of polymorphic loci which potentially can generate a significant $\mathrm{D}$ value. Nothing is known of linkage, which could have reduced the relevant total number of combinations. However, the distribution of $\mathrm{D}$ values among loci is far from random. $M e-2$ participates in 40 per cent of all significant D values. Three additional loci $(A p-1, A p-2$ and $\alpha G p d h)$ each participates in 20 per cent of the significant $D$ values. Therefore, we consider that at least some of the observed $\mathrm{D}$ values are real.

Using climatic and edaphic factors, we ran Spearman rank correlations on the number of significant D values in each of the 14 populations, with complete ecological data, and found nearly significant correlations between the number of significant $\mathrm{D}$ values and with the number of rainy days $\left[r_{\mathrm{s}}=-0.494(P<0.10)\right]$, soil type, loess $\left[r_{s}=0.478(P<0.10)\right]$, and territory size $\left[r_{\mathrm{s}}=0.547 \quad(P<0.10)\right]$. The number of the gametic phase disequilibria increases with ecological stresses eastwards and southwards, towards the steppes and desert, even in the small Sede-Boqer and Egyptian populations.

\section{Discussion}

\section{Natural selection and genetic differentiation}

Natural selection in its various forms appears to be a major differentiating and orienting force of evolutionary change in proteins, and also, most likely in DNA polymorphisms (Nevo, 1988a, b, 1991). This conclusion was derived from an extensive research programme conducted at the Institute of Evolution on plants and animals during the last two decades, using the environmental genetic correlation methodology at three geographical levels. These include local (microgeographical), regional (Israel and the entire Near East), and global levels, primarily at the protein level, but now also at the DNA nuclear (Nevo, 1991; Nevo et al., 1992; Ben-Shlomo et al., 1993) and extranuclear levels (Nevo \& Beiles, 1992; Nevo et al., 1993).

\section{Genetic diversity in subterranean mammals}

Subterranean mammals assumed a special role in our studies of genetic diversity. The major reason derived from the study of subterranean mole rats of the $S$. ehrenbergi superspecies in Israel as an evolutionary model of active speciation and adaptive radiation (Nevo, 1991). Later, we extended the S. ehrenbergi evolutionary model, primarily the aspect of genetic diversity and divergence, to all other subterranean and 
fossorial mammals (Nevo et al., 1990, and see generally Nevo \& Reig, 1990). We have compared and contrasted the levels of heterozygosity of subterranean and fossorial mammals to those of similar-sized, small mammals living aboveground. We have clearly substantiated on a large statistical base, involving 184 species, the prediction of the niche-width variation hypothesis (Van Valen, 1965) at the genetic allozymic level, i.e. lower genetic diversity in the narrower subterranean and fossorial niches (Nevo, 1979).

The niche-width genetic-variation hypothesis was recently verified in the subterranean rodents endemic to Africa (Nevo et al., 1987; Honeycutt et al., 1991; Burda et al., 1992). It was likewise verified in the two families of subterranean insectivore moles, the Talpidae (Filippucci et al., 1987; Yates and Moore, 1990) and the endemic African golden moles, the Chrysochloridae (Filippucci et al., 1991). By contrast, several studies demonstrated in some subterranean rodent species high $H$ values, such as in the North American Thomomys bottae (Patton \& Yang, 1977) and Ctenomyidae (Sage et al., 1986). Alternative hypotheses explaining the low $H$ in subterranean mammals suggest that historical bottlenecks or demographical drastic subdivision of small populations caused the reduction in $H$ (e.g., Selander et al., 1974; Patton, 1990; Honeycutt et al., 1991).

\section{Genetic diversity in the S. ehrenbergi superspecies}

In this study we revisited earlier allozyme studies on $S$. ehrenbergi that were conducted on a small number of loci, first analysing 17 loci and 383 individuals at the species level (Nevo \& Shaw, 1972), and then eight loci and 797 individuals comprising 21 populations (Nevo \& Cleve, 1978). By contrast, in the present study we analysed 241 individuals involving nine chromosomal species, four from Turkey, four from Israel and one from Egypt in 23 populations, and we increased the number of loci from a total of 25 studied earlier to 36 here. Also, only 11 of the loci are shared between the previous and the present study.

The results described above indicate an overall higher level of genetic diversity in the four Israeli species $(2 n=52,54,58$ and 60$)$ compared with the previous studies (Nevo \& Shaw, 1972; Nevo \& Cleve, 1978). The mean $H$ for the $S$. ehrenbergi superspecies in Israel as a whole, and for the $2 n=52,54,58$ and 60 species was 0.039 and $0.035,0.016,0.037$ and 0.069 , respectively, in the earlier study (Nevo \& Cleve, 1978); but in this study the overall mean was 0.051 and 0.043 , $0.041,0.074$ and 0.078 , respectively. Notably, while the general levels of $H$ were higher, the general interspecific pattern, and particularly the southward trend of increasing level of heterozygosity with aridity and climatic unpredictability, is retained in this study. Notably, the wider climatic fluctuations in arid regions (expressed by rainfall variation between years) represent a wider temporal niche.

The discrepancy between the earlier and current levels of genetic diversity presumably derive from the following factors: (1) 25 additional loci, mostly polymorphic, were added to the analysis, while seven blood proteins, mostly monomorphic, were excluded from this analysis; and (2) higher numbers of populations, particularly steppic and desert ones in Israel, including desert isolates, were added to the new analysis.

The results from the study described here may seem to contradict the niche-width genetic-variation hypothesis for subterranean mammals at first, but in fact the opposite is true. The average high $H$ value in the $S$. ehrenbergi superspecies reflects the higher $H$ value in some desert isolates. These reflect the general rule that $H$ is higher in the climatically unpredictable aboveground climatic regions in plants, invertebrates and vertebrates (Nevo \& Beiles, 1988, 1989). This biological rule is also clearly reflected in the underground, relatively more constant and narrow-niche, burrow system of Spalax.

In most cases where high $H$ was reported in subterranean mammals (Patton \& Yang, 1977; Nevo \& Cleve, 1978; Sage et al., 1986) the higher values are primarily derived from populations living in warmer lowlands (e.g. Thomomys bottae displays a high $H$ value compared with $T$. talpoides which lives in the mountains; but see opposite results in intraspecific analysis of $H$ in $T$. bottae, Patton \& Smith, 1990). Higher $H$ values are also derived from populations approaching steppic, more arid and climatically unpredictable environments, such as $S$. ehrenbergi $2 n=58$ and $60, S$. leucodon in Turkey, approaching the central Anatolian Plateau (Nevo et al., 1989, and unpublished data), as well as several species of Ctenomys. The niche-width genetic-variation hypothesis cannot be assessed on the basis of individual species, especially not those living in semi-arid and arid environments, but must rely on a thorough statistical analysis, involving many species, ideally spread over increasing aridity, or generally, climatic unpredictability gradients. Clearly, these results strongly support the environmental selection theory of genetic diversity comprising as a subset the niche-width variation hypothesis.

\section{The environmental selection hypothesis of genetic diversity in subterranean mammals}

Our thorough statistical analysis of mammalian species in general (Nevo, 1985) was later extended (Nevo et al., 
1990) and involved 184 small mammalian species, both insectivores and rodents: 111 living aboveground and 73 species living partly (fossorial, $N=28$ ) or totally (subterranean, $N=45$ ) underground. Our results were clear and statistically significant: both overall, and in insectivores and rodents separately, the narrow-niche fossorial and subterranean species displayed significantly lower values of observed $H$ than small mammalian species living aboveground. This was true in all parametric and non-parametric analyses. Subterranean and fossorial small mammals living in a relatively narrow ecological niche are more homozygous in protein diversity, primarily isozymes, compared with aboveground species. This pattern clearly corroborates the environmental theory of the nichewidth variation hypothesis of genetic diversity, and is also true locally, regionally and globally (Nevo, 1988a).

The paucity of heterozygotes in Spalax populations, as reflected by the deviation from the Hardy-Weinberg expectations (Table 3), suggests the operation of the Wahlund effect in these sedentary low migrating animals. This pattern may be generated by micro divergence selecting different homozygotes in different microhabitats.

The evidence presented here for $S$. ehrenbergi strongly supports the ecological determinants of genetic diversity and differentiation. First, Spalax populations in Israel showed in a thorough census across the country, the following results (Nevo et al., 1982). (1) The overall but underestimated number of mole rats, in their range of $15,500 \mathrm{~km}^{2}$ in Israel, amounts to 1.6-2 million individuals. (2) The number of animals per $\mathrm{km}^{2}$ for $2 n=52,54,58$ and 60 species was $140,177,101$ and 91, respectively, decreasing southward towards the desert. (3) Populations are largely continuously distributed in their main ranges corresponding to an isolation by distance model, but become semi-isolated and isolated in their marginal peripheries. These results in the Israeli $S$. ehrenbergi superspecies contrast with the commonly held view that the population structure of subterranean mammals always involves numerous geographically partially isolated demes across their ranges (e.g. Patton, 1990). Moreover, they suggest that clinal differentiation characterizes the main distributional ranges climaxing in the highest levels of $H$ in the south.

Can alternative hypotheses, including historical, demographical, gene flow and inbreeding models (e.g. Smith et al., 1975; Patton \& Yang, 1977; Patton, 1990; Honeycutt et al., 1991) explain the general pattern of relatively lower $H$ in subterranean mammals? While clearly some or all of these theories may partly explain the patterns unravelled, they are not able to explain the increase in genetic diversity southwards towards the desert. (1) The steppic and desert climates are more unpredictable and variable over time (Atlas of Israel, 1970), thus exposing a broader climatic niche than that of the mesic northern populations. (2) The southern populations are lower in density (Nevo et al., 1982), and are liable more to bottlenecking and stochastic processes (genetic drift) in general. Small population size, drift and bottlenecking are expected to reduce the frequency of heterozygotes. Therefore, the higher intrapopulation genetic diversity in the south negates all predictions of neutral and historical hypotheses that suggest a decrease in $H$ in the smaller southern populations.

We submit that ecological factors are predominant in shaping genetic diversity patterns in nature. This is clearly substantiated by the data presented in this paper, but was earlier corroborated on all scales (Nevo, $1988 \mathrm{a}, \mathrm{b})$, involving local microgeographical scales in several species of animals and plants, as well as macrogeographically on the regional scale of Israel, involving 21 unrelated species of plants and animals (Nevo \& Beiles, 1988, 1989), and the global scale involving 1111 species of plants and animals across all life zones (from the arctic through the temperate to the tropic), and through diverse habitats and climates (Nevo et al., 1984). It was also demonstrated recently on a global scale in 189 amphibian species (Nevo \& Beiles, 1991).

\section{Peripheral small isolates and speciation}

Speciation may primarily originate in the peripheral small isolates, where the initial fixation of spontaneous chromosomal mutations may take place by random genetic drift, but where sufficient genetic diversity exists, amounting to the highest values of $H$ across the range (Nevo, 1989). Completion of speciation involves colonization of the new homozygous chromosome form into the ecological vacant niche in which it is adaptively superior to its ancestor.

The ideas of peripatric speciation mentioned above and elaborated by Nevo (1989) are supported by our present results concerning allozymes, as well as by RFLP results overviewed by Nevo (1991). The species phylogenetic tree and evolutionary divergence times are similar to those derived from other pieces of molecular evidence such as DNA hybridization (Catzeflis et al., 1989) and mtDNA (Nevo \& Beiles, 1992; Nevo et al., 1993).

Genetic diversity in the isolates and semi-isolates, at the allozyme level described here, at the nuclear DNA level (Nevo, 1991) and the extranuclear, mitochondrial level (Nevo \& Beiles, 1992; Nevo et al., 1993) are remarkably and unexpectedly higher than any expectations based on history, demography, gene flow or 
inbreeding. All these four explanatory models, individually or in combination, fail to explain our results that indicate high levels of protein and DNA polymorphisms in the small semi-isolated and isolated (as well as in the relatively presumed highly inbred desert isolates) of the species with $2 n=60$. The isolation and smallness (see details in Nevo, 1989) of individuals at Sede-Boqer $(<100)$ imply very low levels of genetic diversity due to the following reasons. The desert isolates do not obtain gene flow from the main range due to the harsh and inhospitable desert that separated them during the 10,000 years of the Holocene. Likewise, their small size reinforces inbreeding even if mate selection is operating. Finally, the entire $2 n=60$ species is in the most recent species pair of the entire complex. As such, it would be expected to harbour less genetic diversity. In fact it, and especially its smaller peripheral isolates, defy all theoretical expectations based on history and demography, and strongly suggest the operation of natural selection strong enough to overcome $N_{e} \leq 100$, in these desert isolates, in accordance with the environmental selection theory of genetic diversity and its niche-width genetic-variation subhypothesis.

\section{Conclusions}

Natural selection appears to be the major evolutionary force shaping the levels and differentiation of genetic diversity of the $S$. ehrenbergi superspecies based on the following reasoning.

(1) Genetic diversity within populations and species is non-random in both space and time as indicated by the patterns of allele frequencies, genetic indices, environmental correlates, and gametic phase disequilibria.

(2) Natural selection is operating not only on single genes but also on two-locus combinations (gametic phase disequilibria) and multilocus structures (heterozygosity).

(3) There is a low rate of migration as indicated by the spatial autocorrelation analysis.

(4) The high levels of genetic diversity in the small peripheral isolates negates any model other than natural selection.

We conclude that at the molecular genetic level (of proteins described here), the nuclear and mitochondrial DNA level (see Nevo, 1991; Nevo \& Beiles, 1992; Nevo et al., 1993) and the organismic level (Nevo, 1991), ecology plays a major role in adaptive radiation and speciation in mole rats.

\section{Acknowledgements}

We thank the Israeli Discount Bank Chair of Evolutionary Biology, and the Ancell-Teicher Research Foundation for Genetics and Molecular Evolution established by Florence and Theodore Baumritter of New York, for financial support of this research.

\section{References}

ATLAS OF ISRAEL. 1970. Surveys of Israel. Ministry of Labour, Jerusalem and Elsevier, Amsterdam.

BEN-SHLOMO, R., SHIN, H-S. AND NEVO, E. 1993. Period-homologous sequence polymorphisms in subterranean mammals of the Spalax ehrenbergi superspecies in Israel. Heredity, 70, 111-121.

BURDA, H., FILIPPUCCI, M. G., MACHOLAN, M., NEVO, E. AND ZIMA, J. 1992. Biological, allozyme, and karyotype differentiation of African mole-rats (Cryptomys, Bathyergidae) from Zambia. Z. Saugetierk. Suppl., 57, 11-12.

CATZEFLIS, F. M., NEVO, E., AHLQUIST, J. E. AND SIBLEY, C. G. 1989. Relationships of the chromosomal species in the Eurasian mole rats of the Spalax ehrenbergi group as determined by DNA-DNA hybridization, and an estimate of the spalacid-murid divergence time. J. Mol. Evol., 29, 223-232.

FELSENSTEIN, J. 1991. PHYLIP (Phylogeny Inference Package) manual of Version 3.4. Distributed by the author. University of Washington, Seattle.

FILIPPUCCI, M. G., NASCETTI, G., CAPANNA, E. AND BULLINI, L. 1987. Allozyme variation and systematics of European moles of the genus Talpa (Mammalia, Insectivora). J. Mammal., 68, 487-499.

FILIPPUCCI, M. G., HICKMAN, G. C., CAPANNA, E. AND NEVO, E. 1991. Genetic diversity and differentiation of the endemic subterranean golden moles of South Africa (Chrysochloridae, Mammalia). Biochem. Syst. Ecol., 19, 461-466.

GILlespie, J. 1991. The Causes of Molecular Evolution. Oxford University Press, New York.

GORMAN, G. C. AND RENZI, J., JR. 1979. Genetic distance and heterozygosity estimates in electrophoretic studies: effects of sample size. Copeia, 1979, 242-249.

HONEYCUTT, R. L., NELSON, K., SCHLLITER, D. A. AND SHERMAN, P. W. 1991. Genetic variation within and among populations of the Naked mole-rat: Evidence from nuclear and mitochondrial genomes. In: Sherman, P. W., Jarvis, J. U. M. and Alexander, R. D. (eds) The Biology of the Naked Mole-Rat, pp. 195-208. Princeton University Press, Princeton, NJ.

KIMURA, M. 1983. The Neutral Theory of Molecular Evolution. Cambridge University Press, Cambridge.

NEI, M. 1971. Interspecific gene differences and evolutionary time estimated from electrophoretic data on protein identity. Am. Nat., 105, 385-398.

NEI, M. 1972. Genetic distance between populations. Am. Nat., 106, 283-292.

NEI, M. 1973. Analysis of gene diversity in subdivided populations. Proc. Natl. Acad. Sci. U.S.A., 70, 3321-3323. 
NEI, M. 1978. Estimation of average heterozygosity and genetic distance from a small number of individuals. Genetics, 89, 583-590.

NEvo, E. 1978. Genetic variation in natural populations: Patterns and theory. Theor. Pop. Biol., 13, 121-177.

NEVO, E. 1979. Adaptive convergence and divergence of subterranean mammals. Ann. Rev. Ecol. Syst., 10, 269-308.

NEVO, E. 1983. Population genetics and ecology: the interface. In: Bendall, D. S. (ed.) Evolution from Molecules to Men, pp. 287-321. Cambridge University Press, Cambridge.

NEVO, E. 1985. Ecological and populational correlates of allozyme polymorphisms in mammals. Acta Zool. Fenn., 170, 25-29.

NEI, M. 1987. Molecular Evolutionary Genetics. Columbia University Press, New York.

NEvo, E. 1988a. Genetic diversity in nature: Patterns and theory. Evol. Biol., 23, 217-246.

NEVO, E. 1988b. Natural selection in action: The interface of ecology and genetics in adaptation and speciation at the molecular and organismal levels. In: Tchernov, E. and Yom-Tov, Y. (eds) Zoogeography of Israel, pp. 411-438. Dr. Junk Publications, Holland.

NEVO, E. 1989. Modes of speciation: The nature and role of peripheral isolates in the origin of species. In: Giddings, L. V., Kaneshiro, K. Y. and Anderson, W. W. (eds) Genetics, Speciation and the Founder Principle, pp. 205-236. Oxford University Press, Oxford.

NEVO, E. 1991. Evolutionary theory and processes of active speciation and adaptive radiation in subterranean mole rats; Spalax ehrenbergi superspecies, in Israel. Evol. Biol., 25, 1-125.

NEVO, E. AND SHAW, C. R. 1972. Genetic variation in a subterranean mammal, Spalax ehrenbergi. Biochem. Genet., 7, 235-241.

NEVO, E. AND CLEVE, H. 1978. Genetic differentiation during speciation. Nature, 275, 125-126.

NEVO, E. AND BEILES, A. 1988. Genetic parallelism of protein polymorphism in nature: ecological test of the neutral theory of molecular evolution. Biol. J. Linn. Soc., 35, 229-245.

NEVO, E. AND BEILES, A. 1989. Genetic diversity in the desert: Patterns and testable hypotheses. J. Arid Env., 17, 241-244.

NEVO, E. AND REIG, O. A. (EDS) 1990. Evolution of Subterranean Mammals at the Organismal and Molecular Levels. Alan R. Liss, Inc., New York.

NEVO, E. AND BEILES, A. 1991. Genetic diversity and ecological heterogeneity in amphibian evolution. Copeia, 3, 565-592.

NEVO, E. AND BEILES, A. 1992. MtDNA polymorphisms: evolutionary significance in adaptation and speciation of subterranean mole rats. Biol. J. Linn. Soc., 47, 385-405.

NEVO, E., KIM, Y. I., SHAW, C. R. AND THAELER, C. S., Jr. 1974. Genetic variation, selection and speciation in Thomomys talpoides pocket gophers. Evolution, 28, 1-23.

NEVO, E., HETH, G. AND BEILES, A. 1982. Population structure and evolution in subterranean mole rats. Evolution, 36, 1283-1289.
NEVO, E., BEILES, A. AND BEN-SHLOMO, R. 1984. The evolutionary significance of genetic diversity: ecological, demographic and life history correlates. In: Mani, G. S. (ed.) Evolutionary Dynamics of Genetic Diversity. Lect. Notes Biomath., 53, 13-213.

NEVO, E., CORTI, M., BEN-SHLOMO, R., BEILES, A., JARVIS, J. U. M. AND HICKMAN, G. C. 1985a. Karyotype and allozyme differentiation in the endemic subterranean mole rats of Africa, the Bathyergidae. In: Abstr. 4th Int. Ther. Congr., Edmonton, Alberta, p. 460.

NEVO, E., BEILES, A. AND BEN-SHLOMO, R. 1985b. Genetic diversity and differentiation in mammals. In: Abstr. 4th Int. Ther. Congr., Edmonton, Alberta, p. 459.

NEVO, E., BEN-SHLOMO, R., BEILES, A., JARVIS, J. U. M. AND HICKMAN, G. C. 1987. Allozyme differentiation and systematics of the endemic subterranean mole rats of South Africa (Rodentia, Bathyergidae). Biochem. Syst. Ecol., 15, 489-502.

NEVo, E., FILIPPUCCI, M. G., SIMSON, S. AND HETH, G. 1989. Karyotype and allozyme differentiation in the Spalax leucodon superspecies from Turkey. In: Abstr. 5th Int. Ther. Congr., Rome, p. 26.

NEVo, E., FILIPPUCCI, M. G. AND BEILES, A. 1990. Genetic diversity and its ecological correlates in nature: Comparisons between subterranean, fossorial, and aboveground small mammals. In: Nevo, E. and Reig, O. A. (eds) Evolution of Subterranean Mammals at the Organismal and Molecular Levels, pp. 347-366. Alan R. Liss, Inc., New York.

NEVO, E., SIMSON, S., HETH, G., REDI, C. AND FILIPPUCCI, M. G. 1991. Recent speciation of subterranean mole rats of the Spalax ehrenbergi superspecies in the El-Hammam isolate, northern Egypt. 6th International Colloquium on the Ecology and Taxonomy of Small African Mammals, Mitzpe Ramon, Israel, p. 43.

NEVO, E., BEN-SHLOMO, R., BEILES, A., HART, C. P. AND RUDDLE, F. H. 1992. Homeobox DNA polymorphisms (RFLPs) in subterranean mammals of the Spalax ehrenbergi superspecies in Israel: Patterns, correlates and evolutionary significance. J. Exp. Zool., 263, 430-441.

NEVO, E., HONEYCUTT, R. L., YONEKAWA, H., NELSON, K. AND HANZAWA, N. 1993. Mitochondrial DNA polymorphisms in subterranean mole rats of the Spalax ehrenbergi superspecies in Israel and its peripheral isolates. Mol. Biol. Evol., 10, 590-604.

PATTON, J. L. 1990. Geomyid evolution: The historical, selective, and random basis for divergence patterns within and among species. In: Nevo, E. and Reig, O. A. (eds) Evolution of Subterranean Mammals at the Organismal and Molecular Levels, pp. 49-69. Alan R. Liss, Inc., New York.

PATTON, J. L. AND YANG, S. Y. 1977. Genetic variation in Thomomys bottae pocket gophers: Macrogeographic patterns. Evolution, 31, 697-720.

PATTON, J. L. AND SMITH, M. F. 1990. The evolutionary dynamics of Thomomys bottae pocket gophers in California. Calif. Univ. Publ. Zool., 123, 1-161.

SAGE, R. D., CONTRERAS, J. R., ROIG, v. G. AND PATTON, J. L. 1986. Genetic variation in the South American burrowing 
rodents of the genus Ctenomys (Rodentia: Ctenomyidae). Z. Saugetierkunde, 51, 158-172.

SAITOU, N. AND NEI, M. 1987. The Neighbor-Joining method: A new method for reconstructing phylogenetic trees. Mol. Biol. Evol., 4, 406-425.

SARICH, V. M. 1977. Rates, sample sizes and the neutrality hypothesis for electrophoresis in evolutionary studies. Nature, 263, 24-28.

SAVIC, 1. AND NEVo, E. 1990. The Spalacidae: Evolutionary history, speciation, and population biology. In: Nevo, E. and Reig, A. O. (eds) Evolution of Subterranean Mammals at the Organismal and Molecular Levels, pp. 129-153. Alan R. Liss, Inc., New York.

SELANDER, R. K., KAUFMAN, D. W., BAKER, R. J. AND WILLIAMS, S. L. 1974. Genic and chromosomal differentiation in pocket gophers of the Geomys bursarius group. Evolution, 28, 557-564.

SMITH, M. H., CHARLES, T. G., Jr. AND RAMSEY, P. R. 1975. Genic heterozygosity and population dynamics in small mammals. In: Markert, C. L. (ed.) Isozymes IV, Genetics and Evolution, pp. 85-102. Academic Press, Inc., San Francisco.

SOKAL, R. R. AND ODEN, N. L. 1978a. Spatial autocorrelation in biology. I. Methodology. Biol. J. Linn. Soc., 10, 199-228.
SOKAL, R. R. AND ODEN, N. L. 1978b. Spatial autocorrelation in biology. II. Some biological implications and four applications of evolutionary and ecological interest. Biol. J. Linn. Soc., 10, 229-249.

SOKAL, R. R. AND WARTENBERG, D. w. 1983. A test of spatial autocorrelation using an isolation-by-distance model. Genetics, 105, 219-237.

SOULE, M. AND STEWART, B. R. 1970. The "niche variation" hypothesis: a test and alternatives. Am. Nat., 104, 85-97.

sPSs, 1990. sPSS Reference Guide, Release 4, sPSs Inc., Chicago. SWOFFORD, D. L. AND SELANDER, R. B. 1989. Manual of BIOSYS-1 Computer Program, Release 1.7. Distributed by D. L. Swofford, Illionoi's Natural History Survey.

VAN VALEN, L. 1965. Morphological variation and width of ecological niche. Am. Nat., 99, 377-390.

VITHAYASAI, C. 1973. Exact critical values of the Hardy-Weinberg test statistic for two alleles. Commun. Stat., 1, 229-242.

YATES, T. L. AND MOORE, D. w. 1990. Speciation and evolution in the family Talpidae (Mammalia: Insectivora). In: Nevo, E. and Reig, O. A. (eds) Evolution of Subterranean Mammals at the Organismal and Molecular Levels, pp. 1-22. Alan R. Liss, Inc., New York. 